\title{
Review Article \\ Molecular, Phenotypic Aspects and Therapeutic Horizons of Rare Genetic Bone Disorders
}

\author{
Taha Faruqi, ${ }^{1}$ Naveen Dhawan, ${ }^{1}$ Jaya Bahl, ${ }^{2}$ Vineet Gupta, ${ }^{3}$ Shivani Vohra, \\ Khin Tu, ${ }^{1}$ and Samir M. Abdelmagid ${ }^{5}$ \\ ${ }^{1}$ Nova Southeastern University Health Sciences Division, Fort-Lauderdale-Davie, FL 33314, USA \\ ${ }^{2}$ Florida International University (FIU), Miami, FL 33174, USA \\ ${ }^{3}$ Department of Medicine, University of California San Diego (UCSD), 200 West Arbor Drive, MC 8485, San Diego, CA 92103, USA \\ ${ }^{4}$ University of Pennsylvania School of Dental Medicine, Philadelphia, PA 19104, USA \\ ${ }^{5}$ Northeast Ohio Medical University (NEOMED) School of Medicine, Rootstown, OH 44272, USA \\ Correspondence should be addressed to Vineet Gupta; vineetgsvm@gmail.com
}

Received 28 February 2014; Revised 12 August 2014; Accepted 24 August 2014; Published 22 October 2014

Academic Editor: Vasiliki Galani

Copyright (c) 2014 Taha Faruqi et al. This is an open access article distributed under the Creative Commons Attribution License, which permits unrestricted use, distribution, and reproduction in any medium, provided the original work is properly cited.

\begin{abstract}
A rare disease afflicts less than 200,000 individuals, according to the National Organization for Rare Diseases (NORD) of the United States. Over 6,000 rare disorders affect approximately 1 in 10 Americans. Rare genetic bone disorders remain the major causes of disability in US patients. These rare bone disorders also represent a therapeutic challenge for clinicians, due to lack of understanding of underlying mechanisms. This systematic review explored current literature on therapeutic directions for the following rare genetic bone disorders: fibrous dysplasia, Gorham-Stout syndrome, fibrodysplasia ossificans progressiva, melorheostosis, multiple hereditary exostosis, osteogenesis imperfecta, craniometaphyseal dysplasia, achondroplasia, and hypophosphatasia. The disease mechanisms of Gorham-Stout disease, melorheostosis, and multiple hereditary exostosis are not fully elucidated. Inhibitors of the ACVR1/ALK2 pathway may serve as possible therapeutic intervention for FOP. The use of bisphosphonates and IL-6 inhibitors has been explored to be useful in the treatment of fibrous dysplasia, but more research is warranted. Cell therapy, bisphosphonate polytherapy, and human growth hormone may avert the pathology in osteogenesis imperfecta, but further studies are needed. There are still no current effective treatments for these bone disorders; however, significant promising advances in therapeutic modalities were developed that will limit patient suffering and treat their skeletal disabilities.
\end{abstract}

\section{Introduction}

In the spectrum of orthopaedic diseases, rare genetic bone disorders are often ignored as major diseases such as osteoporosis generally attract more research funding and attention from the research community. A rare disease is defined as one affecting less than 200,000 individuals, according to the US National Organization of Rare Diseases (NORD). Rare bone disorders remain a serious problem in orthopaedics and result in significant morbidity and mortality in patients around the world.

Often a primary problem with rare bone diseases remains to be a lack of understanding of the underlying mechanism. Yet, in recent years many advances have occurred that are promising for the prospect of finding cures. In 2006, the gene for fibrodysplasia ossificans progressiva (FOP) was identified by researchers at the University of Pennsylvania, marking a significant milestone in the understanding of this disease. Prior to this, its etiology remained elusive. While this does not in and of itself translate to a cure, the discovery provides direction for researchers to investigate possible points of disruption of the basic pathway of FOP. Yet, other rare disorders still remain mysteries.

This review summarizes the most current trends in the search for therapeutic interventions for nine rare bone disorders: fibrous dysplasia, Gorham-Stout syndrome, fibrodysplasia ossificans progressiva, melorheostosis, multiple hereditary exostosis, osteogenesis imperfecta, and craniometaphyseal dysplasia. 


\section{Fibrous Dysplasia}

Fibrous dysplasia (FD) is a rare bone disease characterized by replacement of the medullary cavity with fibrous tissue. Any region of the skeleton can be affected by FD, where the most common areas involved include facial bones, the tibia, femur, and the ribs [1]. Several forms of FD exist. The monostotic form of FD is limited to one bone, whereas the polyostotic form is manifest in multiple bones [2]. McCune-Albright syndrome is another variant of FD and, in addition to bone involvement, is associated with endocrine dysfunctions such as Cushing syndrome, hyperthyroidism, and acromegaly [1, 2]. FD causes chronic pain in patients due to bone overgrowth. Other long term problems include bony deformities, unequal limb lengths, and diminished bone strength leading to a high risk of fractures.

FD displays no predilection for either gender. The monostotic form is more prevalent than the polyostotic form, with the variants occurring at a ratio of $7: 3$, respectively [3]. The monostotic form classically occurs in individuals in their 20 s to 30 s whereas the polyostotic form is usually seen in children. Polyostotic FD usually enters dormancy at the onset of puberty, but pregnancy may result in reactivation of the disease [1].

FD results of mutations in the guanine nucleotide binding, alpha stimulating (GNAS) complex locus, located on chromosome 20 [4]. The mutations occur postzygotically and lead to constitutive activation of $\mathrm{G} \alpha_{\mathrm{s}}$, resulting in stimulation of the Wnt/ $\beta$-catenin signaling pathway $[4,5]$. Mutation activation of $\mathrm{G} \alpha$ s subunit leads to high levels of cyclic adenosine monophosphate (cAMP) levels that mediate the downstream functions in the affected cells. In particular, the transcription factors cFos and cJun and the cytokine IL-6 are upregulated in osteoclasts, resulting in excessive bone resorption and dysplastic fibrous growth $[1,6]$.

Recent study showed that transgenic mice with constitutive expression of the $\mathrm{G} \alpha$ s subunit developed an inherited pathologically replication of human FD. The characteristic FD lesions in mice developed only in postnatal life as in human FD [6]. In the affected bone, the lesions develop through a sequence of three consecutive stages: a primary modeling phase characterized by excess medullary bone formation; a secondary phase, with excess, inappropriate remodeling; and a tertiary phase of fibrous dysplastic in the marrow cavity that replicates the human bone pathology in mice of more than 1 year old $[6,7]$.

$\mathrm{X}$-ray diagnostic features of FD are a characteristic hazy bone lesion (ground glass). For most parts, this radiologic entity is sufficient for the initial diagnosis of the disease. However, in patients where metastasis may pose a viable concern, a PET/CT may be considered. However, Su et al. concluded that this alone may not be enough [8]. They conducted Ffluoro-2-deoxy-glucose positron emission tomography (FFDG PET/CT) on a female patient in whom breast cancer recurrence was suspected. FD was an incidental finding on PET/CT. However, they noted that the dysplastic lesion mimicked metastasis. MRI proved to be a useful modality in differentiating FD from metastasis. Other novel approaches of detecting the disease are also being pursued. TabareauDelalande et al. [9] demonstrated that GNAS mutations are specific for fibrous dysplasia among other fibroossifying lesions. Thus, DNA markers for the GNAS mutation may provide an alternate means of diagnosing the disease in more complicated cases of FD.

In the present, there is no cure for FD and the management is composed largely of reduction of pain, preventing further degeneration of bone, and surgical intervention to reshape and restore the functionality of the affected bone. A current approach that aims at both strengthening bone and reducing pain is bisphosphonate therapy. Mäkitie et al. [10] administered bisphosphonates intravenously in a patient with mandibular FD. The therapeutic approach resulted in rapid reduction of pain, stabilized turnover of bone, and even proved to be cosmetically beneficial. In patients that are nonresponsive to bisphosphonates, Chapurlat et al. [11] suggested the use of IL-6 inhibitors such as tocilizumab, a monoclonal antibody used to treat rheumatoid arthritis (RA). A study investigating the effect of tocilizumab on systemic bone resorption through tracking serum cross-linked Cterminal telopeptide of type I collagen (CTX and ICTP) revealed a significant decrease in bone resorption with the therapy [12]. Therefore, this approach could also be useful in preventing the bone resorption seen in FD.

Several potential therapeutic interventions may be employed (Figure 1). A possible therapeutic strategy to be pursued in the future could be targeting the Wnt/ $\beta$ catenin pathway. If the Wnt signaling pathway is halted, $\beta$-catenin will not accumulate within the cell since it is marked for ubiquitination by casein kinase $1 \alpha(\mathrm{CK} 1 \alpha)$, protein phosphatase 2A (PP2A) adenomatosis polyposis coli (APC), Axin, and glycogen synthase kinase 3 (GSK3) [13]. Ubiquitination of $\beta$-catenin would lead to its proteasomal degradation, thus preventing it from eliciting a cellular response contributing to FD. Therefore, if Wnt proteins can be selectively bound by ligand analogs and inactivated, the tumorigenic fibrous growth will be diminished (Figure 2).

\section{Gorham-Stout Disease}

Gorham's disease (GD), also known as vanishing bone disease, is a rare genetic disorder characterized by bone resorption and localized lymphangiogenic proliferation [14]. This lymphatic and vascular proliferation within bone is thought to aid in osteolysis. GD shows no preference for gender or race and occurs more often in children and young adults. Although GD manifests itself as a monostotic or polyostotic disease, it more commonly involves the flat bones that form by intermembranous ossification [15].

Diagnosis of GD is challenging; it is often a diagnosis of exclusion. Other differentials such as endocrinopathies, malignancies, and immunologic, infectious, and metabolic etiologies need to be ruled out before a diagnosis of GD can be made $[15,16]$.

A study conducted by Venkatramani et al. [17] revealed insights about GD manifestations. Of the eight patients (median age at diagnosis was 11.5 years) who were part of the study, seven presented with lymphangiomatous lesions in 


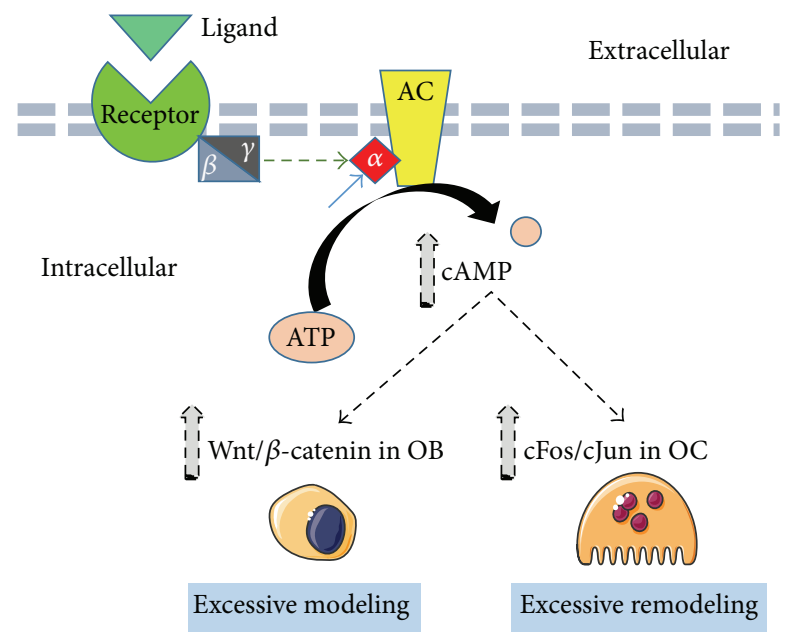

FIGURE 1: Schematic diagram of the pathogenesis of FD: mutation of the $\alpha$ subunit in GNAS (blue arrow) results in autonomous activation of adenylate cyclase (AC) and increased cAMP levels. Cyclic AMP stimulates $\mathrm{Wnt} / \beta$-catenin signaling in osteoblasts leading to excessive bone formation. In addition, cAMP activates cJun and cFos of AP-1 complex in osteoclasts resulting in excessive bone remodeling.

the soft tissues adjacent to the involved bone. This finding is particularly interesting since Bruch-Gerharz et al. [18] also found that skin and soft tissues adjacent to the bone lesions have remarkable lymphatic vascular malformations. Furthermore, the skin and soft tissue involvement preceded bone osteolysis by several years. Therefore, one can conclude that the lymphatic vascular malformations presenting in GD can potentially serve as an early diagnostic sign. BruchGerharz et al. also demonstrated that magnetic resonance imaging (MRI) was essential in characterizing the extent of GD progression by tracking lymphatic malformation in tissues [18].

The pathogenesis of GD is not well understood and therefore not many therapeutic modalities are currently available. Recent study showed that lymphatic endothelial cells (LECs) and blood endothelial cells (BECs) in addition to macrophages secrete TNF $\alpha$ and IL- 6 that stimulate osteoclast formation with excessive osteolysis [19]. Macrophages produce VEGF-C and -D that stimulate proliferation of LECs and BECs. Moreover, macrophages produce VEGF-A, -C, and -D and IL-6 that directly stimulate osteoclast differentiation [20] (Figure 3). Furthermore, TNF $\alpha$ secreted by LECs and macrophages inhibits osteoblast differentiation and new bone formation [21]. Devlin et al. [22] demonstrated that the serum from a patient with GD caused increased proliferation of osteoclast-like multinucleated cells when cultured with normal human bone marrow. Furthermore, the levels of IL6 were significantly higher in the serum of GD patients. This suggests that bone resorption observed in GD could be a direct result of increased multinucleated cell activity due to increased IL-6 levels. Therefore, local inhibition of IL-6 production or administration of a drug such as tocilizumab will be beneficial.
Today, there are no set guidelines for the treatment and management of GD. To prevent the production of IL- 6 by proliferating vasculature, radiation therapy, and chemotherapy with interferon $\alpha-2 \mathrm{~b}$ is commonly employed [23], although it is contraindicated in growing children. Different treatment modalities that include surgical resection, arthroplasty, calcitonin, calcium, and vitamin D have been utilized and the results are variable. Bone grafts have also been used, with a debatable successful rate. Hirayama et al. [16] reported that, despite the use of a bone graft, GD recurred in the grafted bone. In a revealing case described by Hammer et al. [24], clinical improvement followed by stabilization of the disease occurred solely after use of low-dose pamidronate therapy. To our knowledge, this is the only known case of a bisphosphonate monotherapy leading to remission of GD (Figure 4).

Other efforts include the identification of diagnostic markers of GD. In a study conducted by Franchi et al. [25], CD105/endoglin, a marker for vascular endothelial cells, was used to assess the nature of the endothelial cells proliferating in GD. CD105 expression was found to be significantly higher in GD vessels compared to those found in osseous hemangioma (\% positive was 58.9 versus 17.2, resp.). Therefore, this marker may offer a potential means of diagnosing patients with GD.

\section{Fibrodysplasia Ossificans Progressiva}

Fibrodysplasia ossificans progressiva (FOP) is a rare devastating autosomal dominant disease that is characterized by heterotrophic ossification ( $\mathrm{HO}$ ) in the soft tissues following a simple injury [26]. The disease affects 1 in 2 million individuals [27]. There are currently about 700 known cases around the world. FOP displays no predilection for gender, race, or geographic location [28]. Although episodic flare-ups occur in FOP, the damage is cumulative, leading to increasing disability. Individuals with FOP display no abnormality at birth, with the exception of congenital great toe malformations [27]. Painful transformation of soft connective tissue into bone begins in the first decade of life [29]. Surgical intervention leads to a sever rebound response marked by rapid bone growth [28].

$\mathrm{HO}$ in FOP is seen initially in the cranial, dorsal, axial, and proximal regions of the body and then later occurs in caudal, ventral, and distal regions. Since there are episodic flare-ups, the disease progression may vary and not follow the previous order in all cases. Skeletal muscles are also involved in the ossification process; however, smooth muscle and cardiac muscle are spared [28]. Kaplan et al. [30] conducted a study to determine the cause of death and lifespan of individuals with FOP. The most common cause of death in FOP was cardiorespiratory failure as a result of thoracic insufficiency syndrome, and the median lifespan of the 371 individuals in the international FOP community was 56 years.

The diagnosis of FOP can be made by the association of progressive ossifying soft tissue swellings and great toe malformations [31]. This association is not often made by clinicians and thus FOP is frequently missed. The affected 


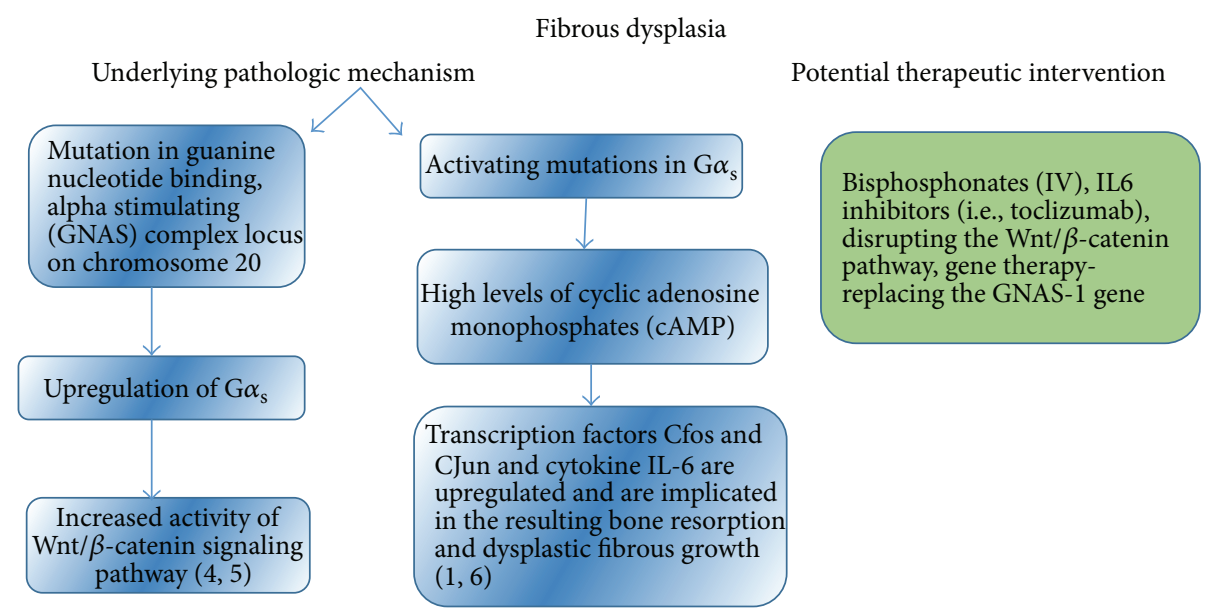

FIGURE 2: Summary of the pathological mechanisms underlying FD and potential therapeutic strategies that may be pursued.

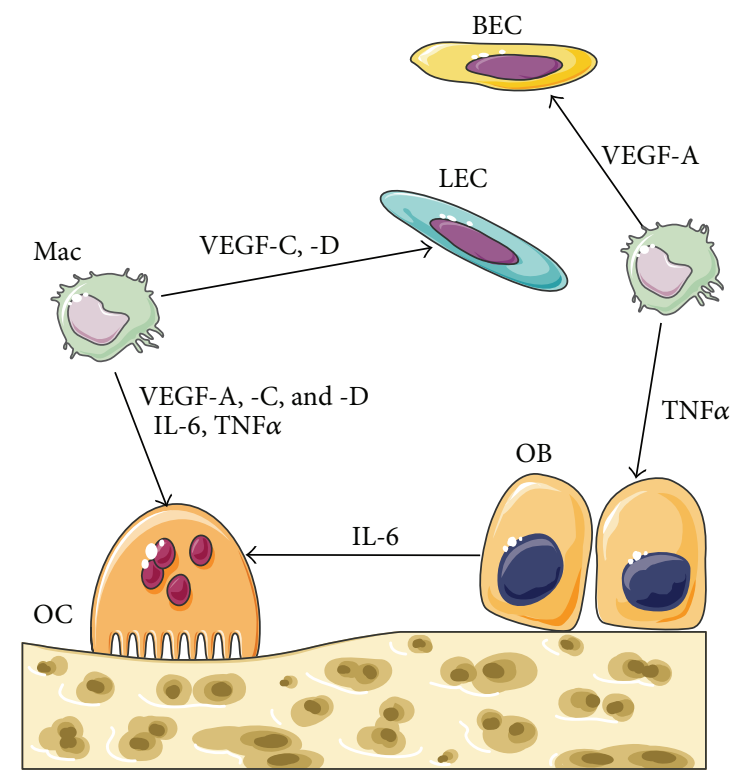

FIGURE 3: Schematic diagram of the pathogenesis of GSD. Lymphatic and blood endothelial cells (LECs), BECs, and macrophages (Mac) secrete $\mathrm{TNF} \alpha$ that stimulate OB to release IL-6. Mac produces VEGF-C and -D that stimulate proliferation of LECs and BECs. Mac also produces VEGF-A, -C, and -D and IL-6 that directly stimulate osteoclast-mediated bone resorption.

individuals are often exposed to unwarranted trauma due to unneeded biopsies of the soft tissue swellings, thereby leading to further exacerbation of the disease.

There is no current cure for FOP. The current management of FOP is early diagnosis, preventing iatrogenic trauma, and alleviating pain during episodic flare-ups. Several studies have indicated that FOP is associated with the bone morphogenetic protein (BMP) signaling pathway. BMPs are responsible for the stimulation of bone formation through binding to the activin receptor type 1 (encoded by the AVCR1 gene receptor), a BMP type 1 receptor. Thus, in 2006 Kaplan et al.
[26] identified a mutation in activin receptor IA/activinlike kinase 2 (AVCR1/ALK2) in all patients presenting with FOP (Figure 5). DNA sequencing displayed the occurrence of missense mutation in the glycine-serine activation domain in individuals with FOP. Not all FOP cases are caused by the common mutation, as there are several FOP variants with varying phenotypes. Importantly, Chakkalakal et al. [32] further elucidated the mechanism of FOP using a FOP knockin mouse model. Thus, FOP results from a mutation in the gene ACVR1/ALK2, which causes the amino acid histidine to be substituted in place of arginine at the 206 codon. Due to the discovery of this highly specific mutation in the FOP gene, therapeutic modalities can now be aimed at blocking the AVCR1/ALK2 pathway. Thus, the identification of factors that are a part of or that aid the BMP signaling pathway has been the focus of recent studies. Mao et al. suggested the potential role of matrix metalloproteinase-10 (MMP-10) in the $\mathrm{HO}$ of muscle in FOP patients. They showed that MMP-10 stimulated myoblast differentiation into osteoblasts through the interactions with BMP pathway [33]. Thus, MMP-10 may serve as a potential therapeutic target. Giacopelli et al. [34] recently reported a significant finding that transcription factors including Egr-1, Egr-2, ZBTB7A/LRF, Heyl, and Sp1 are responsible for the regulation of the ACVR1 promoter through binding to the $-762 /-308$ region. Furthermore, additional studies have shown that miR-148a may be a critical mediatory agent of ACVR1 $[35,36]$. Thus, disruption of the pathway through blocking or slowing down any of these transcription factors presents the most promising form of potential therapy to date.

Importantly, while inhibitors of ALK2 including LDN193189 and dorsomorphin are effective in reducing ALK2 activity, they also block the activity of another BMP receptor, BMPR1 (ALK3) activity [37]. Thus, any viable therapeutic intervention would be one that blocks the hyperactivity of ALK2 without impacting the other kinases in the pathway [33]. Kaplan et al. were able to identify siRNAs which target the ALK2 causing pathology while the normal ALK2 remained unaffected $[37,38]$. Thus, siRNAs from FOP patients have been utilized to retain normal activity of BMP 


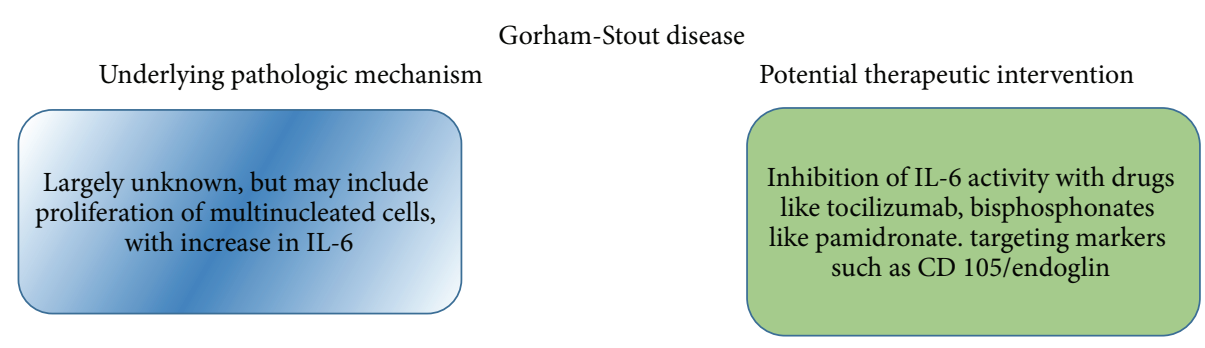

FIGURE 4: Pathogenesis of GD and potential therapeutic interventions.

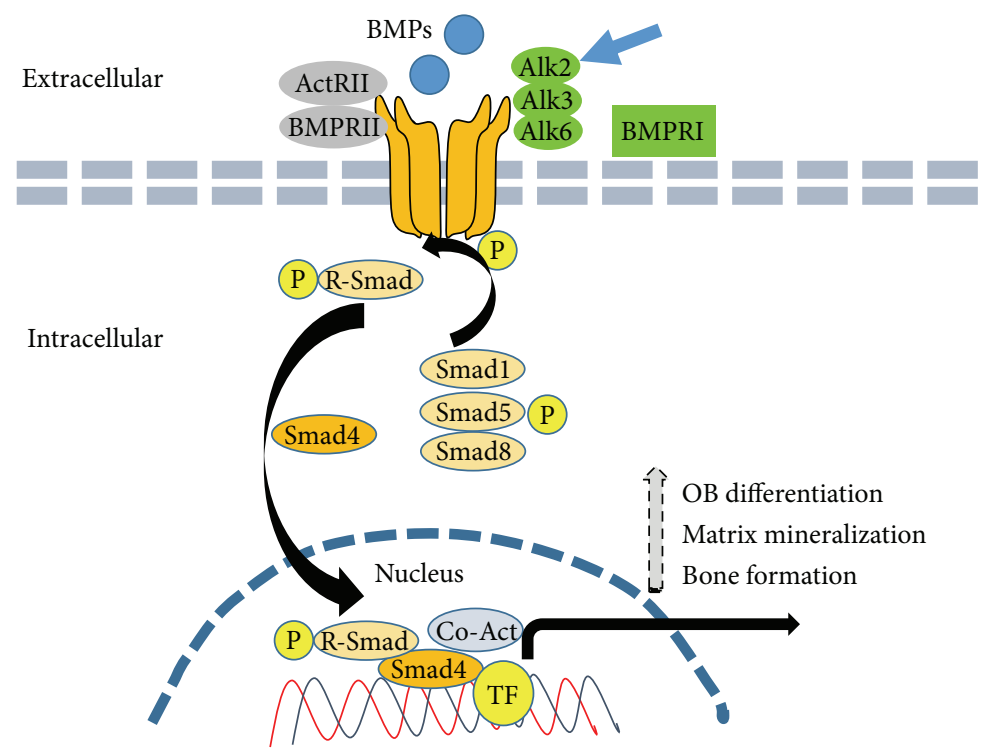

FIGURE 5: Schematic diagram of the pathogenesis of FOB: mutation of the Alk2 subunit (blue arrow) of BMP receptor I leads to constitutive phosphorylation of the downstream regulated-smad1, -5 , and -8 that associate with smad4. Multimeric smad complex translocates to the nucleus and positively regulates several transcription factors responsible for osteoblast differentiation and bone formation.

[37, 38]. Kaplan et al. [38] demonstrated selective suppression of mutated ACVR1 by utilizing ASP-RNAi (allele-specific RNA interference) techniques. This study showed a promising glimpse of the possibility of shutting down ACVR1 activity. Yet, further work is needed to develop an effective regimen of ACVR1 suppression in humans. Figure 6 summarizes the pathogenesis and possible therapeutic strategies that may target FOP.

\section{Melorheostosis}

Melorheostosis is a rare genetic bone disease of unknown etiology in which patients exhibit bone dysplasia marked with benign sclerosis [39]. The disease has no predilection for gender and occurs sporadically. Scleroderma of the skin overlying the affected bone, vascular malformations, and soft tissue masses have also been reported [40]. Spinal sensory nerves are commonly involved [41] and the sclerosis is usually unilateral. The disease can be monostotic and polyostotic or only involve one limb (monomelic) [42]. Involvement of the lower limbs is more commonly seen whereas skull involvement is rare [42]. Histological analysis reveals thickening of the cortical bone that is comprised of mature lamellar and woven bone with adjacent fibrocartilage surrounding coronoid islands $[43,44]$.

The classic radiologic appearance of melorheostosis is "flowing hyperostosis" similar to hardened wax dripped on the side of a candle [41]. As such, upon classic presentation of the disease, diagnosis can be made by $\mathrm{X}$-ray studies followed by increased uptake of radionuclide [41, 45]. The diagnosis can be confirmed by MRI and CT by detecting hyperostosis. Furthermore, MRI can also be used to determine the degree of soft tissue involvement [41]. However, Hollick et al. [45] noted that a milder presentation of melorheostosis may be more challenging to diagnose due to periosteal osteosarcoma and myositis ossificans competing as viable differentials.

There is no treatment for melorheostosis, although several potential therapeutic modalities have been suggested (Figure 7). Current management is highly individualized and is based on the severity of the disease, areas of skeletal involvement, and symptoms experienced by the patient. Surgical treatment is undertaken when an adverse or life threatening complication needs to be avoided. Zeiller et al. [41] performed cervicothoracic decompressive laminectomy to alleviate the 
Fibrousdysplasia ossificans progressiva

Underlying pathologic mechanism

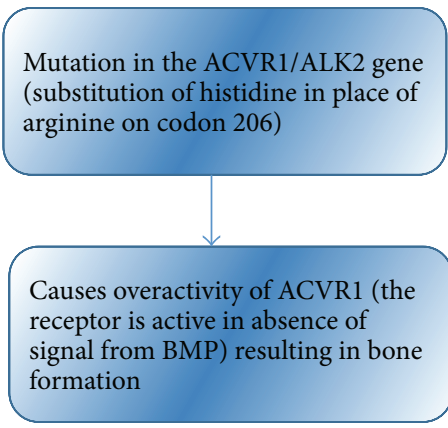

Potential therapeutic intervention

Development of kinase inhibitors that can block ACVR1 or disrupt activity of ACVR1/ALK2 pathway. Inhibitors of ALK2 include LDN193189 and dorsomorphin

FIGURE 6: Summary of the pathogenesis of FOP and potential therapeutic interventions.

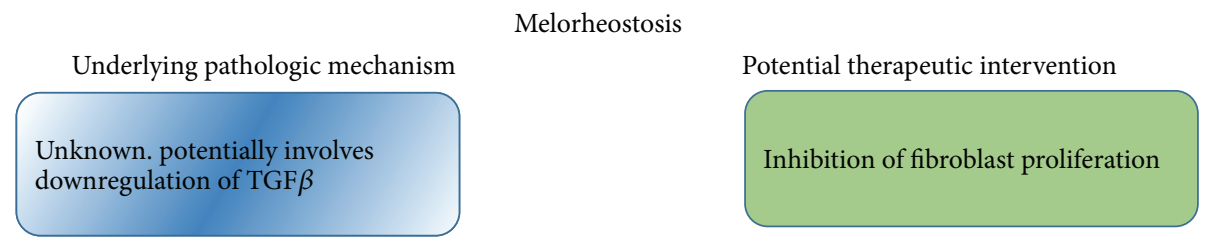

FIgURE 7: Pathogenesis and potential therapeutic interventions of melorheostosis.

worsening neurologic condition in their patients. A follow-up examination conducted six months after the surgery revealed symptomatic improvement of the disease. In another case, Moulder and Marsh [46] were successfully able to treat melorheostosis by total knee arthroplasty. Recently, Hollick et al. [45] were able to achieve a significant reduction of the lesions in melorheostosis with the associated symptoms by a single $5 \mathrm{mg}$ infusion of zoledronic acid administered over a duration of 30 minutes. A follow-up conducted eighteen months after the initial therapy revealed an asymptomatic patient with no further need for treatment.

Hellemans et al. [47] initially linked the etiology of melorheostosis (along with osteopoikilosis and BuschkeOllendorff syndrome) to mutations in the LEMD3 gene. However, in a later study conducted by Hellemans et al. [48], no LEMD3 mutations were identified in patients presenting solely with sporadic melorheostosis. Due to this discovery, the etiology of melorheostosis remains unknown.

Kim et al. [49] found that downregulation of adhesion proteins that regulate osteoblasts, particularly TGF$\beta$ induced gene product, occurs in melorheostosis. They hypothesized that this may be the cause of the presenting hyperostosis and soft tissue abnormalities. Examining the TGF- $\beta$ pathway may provide some clues of the mechanism of melorheostosis. Endo et al. [50] displayed the fact that soft tissue and skin changes occurred due to increased secretion of collagen from fibroblasts. In addition, they proposed that hyperostosis may be responsible for stimulation of fibroblastic secretion. Therefore, inhibition of fibroblast proliferation may lead to an improvement in the soft tissue and skin manifestations of the disease.

\section{Multiple Hereditary Exostosis}

Multiple hereditary exostosis (MHE) is a genetic disorder marked by multiple cartilage-capped boney protuberances (osteochondromas) of the axial skeleton presenting usually before twelve years of age. The usual presentation is unequal limb lengths, reduced range of motion, and osteoarthritis [51]. Joints of the upper and lower limb are commonly affected, particularly the humerus, distal femur, and tibia; however, any bone might also be affected [52].

Diagnosis is made, as outlined by Wuyts and Van Hul [53], primarily using radiologic studies. The characteristic radiographic presentation of MHE is an uninterrupted continuation of the bone cortex into the osteochondroma. Additionally, a family history remarkable for MHE also aids in diagnosis [53].

Pathogenesis of MHE. The genetic basis of MHE has been identified due to mutations in the exostosin-1, EXT1, and EXT2 genes. These genes are involved in heparan sulfate (HS) chain elongation in the Golgi apparatus [54]. Multiple studies have found a more severe disease presentation in individuals with EXT1 mutations versus those with EXT2 mutations $[55,56]$. Recent study showed that inactivation of EXT1 in mouse chondrocytes leads to the development of osteochondroma with characteristic bone deformities that is almost identical to human MHE [57]. It has been reported that EXT1 function is required for maintenance of normal levels of bone morphogenetic protein (BMP) and Wnt, as well as their target genes [58]. Another study indicated that loss of $\beta$-catenin expression (downstream target of BMP) 


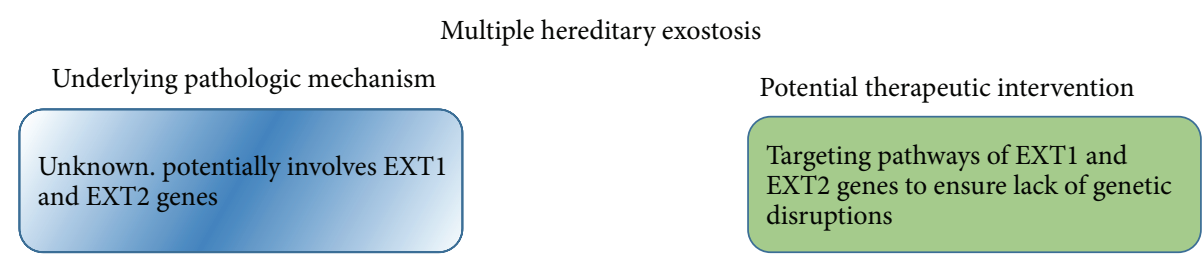

FIGURE 8: Pathogenesis of MHE and potential therapeutic interventions.

in chondrocytes induces periosteal chondroma-like masses, resulting in the cartilage cap in osteochondromas [59].

Since the mutation is known, genetic testing is also currently available for diagnosis of MHE [53]. A novel method of diagnosing MHE has been proposed by AnowerE-Khuda et al. [60]. In their study, they compared HS and chondroitin sulfate (CS) from the serum of MHE patients and healthy individuals. They found that HS was significantly less in the serum of MHE patients and the HS/CS ratios were nearly half those of healthy individuals. Therefore, it was suggested that the HS/CS ratios may be utilized as a diagnostic predictor of MHE.

After diagnosis of MHE, the locations of the lesions, associated symptoms, and any structural deformities and functional limitations need to be documented. If the condition is asymptomatic, no therapy is indicated [53]. Surgeries, when performed, are usually done to limit the presenting symptoms or correct bone defects [61]. Due to undergrowth of the fibula, valgus deformities of the knee and ankle are usually seen [62]. In the upper extremity, the ulna is usually involved in causing radial deformities such as radial head dislocation and radial bowing to occur [63]. Surgical intervention is used in all of these cases.

A serious complication of MHE is malignant transformation into chondrosarcoma [64]. The risk for malignant transformation was previously reported to be 0.6 to $2.8 \%$ [65]. In contrast to this, Kivioja et al. [51] determined higher risk for transformation to chondrosarcoma at $8.3 \%$ in six generations of a family with prevalent MHE. Other literatures, however, reported the risk of malignant transformation as very low [66]. A relatively rare and unique complication that Khan et al. [67] reported in MHE patients was lower extremity ischemia due to popliteal artery occlusion.

Currently, there is no cure for MHE. Although the genetic mutations have been identified, the genetic pathogenesis and particular signaling pathways that lead to the manifestation of the disease remain unknown (Figure 8). If the signaling pathways of EXT1 and EXT2 can be understood, molecular biology can potentially be utilized to alleviate the genetic disturbances due to lack of functional EXT1 and EXT2 genes.

\section{Osteogenesis Imperfecta}

Osteogenesis imperfecta (OI) is a rare genetic bone disease, characterized by the high incidence of fractures with or without minor trauma [68]. Hearing loss is a more commonly observed symptom of OI in older patients. Other classic features, seen in patients with OI, include blue sclerae and triangular facies.
Pathogenesis of OI. Type I collagen is an extracellular matrix protein, mainly found in bone and skin [69]. Two important steps of posttranslational modifications occur: first, hydroxylation of lysine and proline residues that occurs and conveys stability of the collagen triple helix; second, 3-hydroxylation of a proline residue that occurs in the $\alpha$-one chain of type 1 collagen (COL1A1) at position 986 (P986) [69]. In autosomal dominant OI, mutations occur in COL1A1 and COL1A2 that preclude the right folding of type I collagen into proper triple helical structure [69]. Autosomal recessive lethal OI is caused by mutations in cartilage-associated protein CRTAP and prolyl-3-hydroxylase-1 (P3H1, encoded by LEPRE1 gene) which lead to decreased 3-hydroxylation of P986 in type I collagen's $\alpha$-one chain. In both cases, overmodification of type I collagen is noted [69].

A knock-in mouse model for moderately sever OI has been generated $[67,70]$. Characterization of the cellular contribution into the brittle bone disease showed a decrease of the cortical and trabecular bone before and after puberty resulting in 50\% reduction of the bone mass compared to the wild type [70]. Although osteoblasts matrix production was greatly diminished, osteoclast number and activity were increased in the OI mouse compared to the wild type [70]. The study concluded uncoupling between osteoblasts and osteoclasts in brittle bone disease, perhaps due to higher expression of RANK receptors on osteoclast precursors [70]. This cellular imbalance results in decreased bone formation with aging. Interruption of the stimulus that increases osteoclast precursors may leads to new therapeutic modalities for OI. Interestingly, separate study reported the therapeutic benefits of RANKL inhibitors (RANK-Fc) and bisphosphonates in treatment of OI via increased number of bone trabeculae that reduce the incidence of fracture risks [71].

Diagnosis of OI is made based on a history of fractures, family history remarkable for OI, radiographic studies that reveal multiple fractures at different stages of healing, and genetic testing for mutations in COL1A1 and COL1A2. Additionally, biochemical testing of type I collagen may also be conducted. The biochemical testing consists of culturing dermal fibroblasts and analyzing the structure and quantity of the type I collagen produced. Four types of COL1A1 and COL1A2 related OI have been identified (I, II, III, and IV), and biochemical testing has a high sensitivity for detecting these four types of OI [68]. Although the sensitivity of biochemical analysis and genetic testing is comparable, genetic testing is still the recommended first line test for confirmation of OI [72].

Management of the disease is based on the degree of disease progression. Caregivers and parents are advised to 


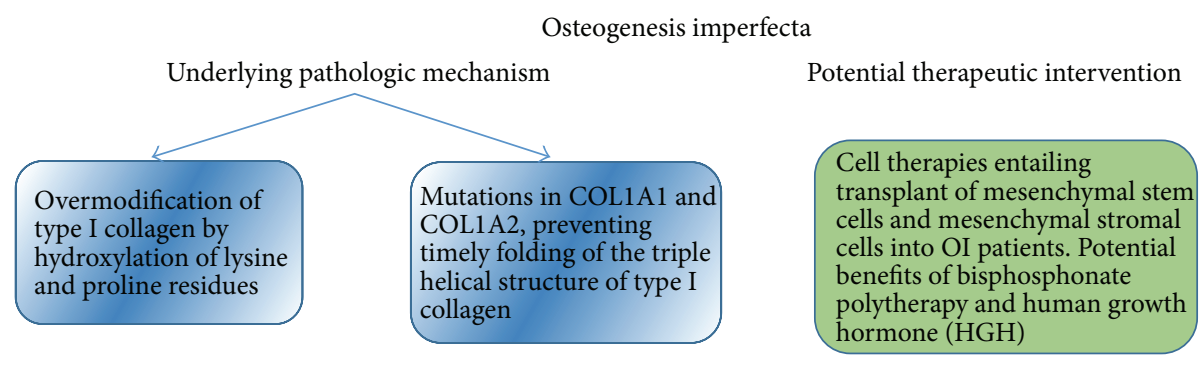

FIGURE 9: OI pathogenesis and potential therapeutic interventions.

handle OI patients safely, since they are susceptible to fractures. As such, management is primarily supportive [73]. Symptomatic surgical interventions include bracing of limbs, stabilization of joints, and reduction of boney deformities [73].

Cases have been reported in which bisphosphonates have been used in an attempt to alter the disease course. Phillipi et al. [74] elaborated the use of bisphosphonates to treat OI. The study indicated that although bone mineral density (BMD) and adult height of patients increased with bisphosphonate therapy, fracture incidence did not decline. This was further confirmed in the study conducted by Sakkers et al. [75], in which the researchers were unable to determine whether the use of olpadronate was able to alter the progression of OI.

Though there is no cure for OI, several therapies are being investigated (Figure 9). A study conducted by Antoniazzi et al. [76] investigated the effects of human growth hormone $(\mathrm{HGH})$ and bisphosphonate polytherapy. The use of growth hormone was correlated with increased BMD and linear growth. Marini et al. [77] conducted a study that yielded similar results. Recently, Otsuru et al. [78] transplanted mesenchymal stem cells and mesenchymal stromal cells into patients with OI. The cell therapies proved to be very effective in this pilot clinical trial. This holds promise for a potential cure for $\mathrm{OI}$ in the near future.

\section{Craniometaphyseal Dysplasia}

Craniometaphyseal dysplasia (CMD) is an extremely rare genetic bone disorder characterized by overgrowth and progressive sclerosis of the craniofacial bones (cranium) and flaring of the metaphyseal plates of femurs (metaphyseal dysplasia) [79, 80]. The lifespan of patients diagnosed with craniometaphyseal dysplasia is normal, except in the most severe cases [81].

The characteristic bone outgrowth in the skull causes many of the symptoms and signs, seen in patients suffering from craniometaphyseal dysplasia. Affected individuals will typically have distinguishing facial features such as thickening of the cranial bones, prominent forehead, paranasal bossing, wide nasal bridge, wide-set eyes (hypertelorism), and a prominent jaw [82]. Infants affected by CMD will have excessive new bone formation (hyperostosis) in their jaw, resulting in delayed teething (dentition) or failure of teeth eruption $[83,84]$. These infants with CMD may also have breathing or feeding problems due to narrow nasal passages.
In the most severe cases, abnormal bone outgrowth can compress the cranial nerves emerging from the brain leading to paralyzed facial muscles (facial nerve palsy), blindness, or deafness $[82,84]$.

Craniometaphyseal dysplasia has two ways of inheritance, the autosomal dominant CMD that is typically more severe than the autosomal recessive form. In most cases this condition is inherited in an autosomal dominant pattern, which means a mutation in one gene copy in each cell is sufficient to cause the CMD disorder $[81,85,86]$. As craniometaphyseal dysplasia runs in families, patients with autosomal dominant CMD typically have one parent who also has the condition. Less often, cases result from new mutations in the gene and occur in people with no history of the disorder in their family. Rarely, craniometaphyseal dysplasia is suspected to have autosomal recessive inheritance when unaffected parents have more than one child with the condition. Autosomal recessive disorders are caused by mutations in both copies of a gene in each cell. The parents of an individual with an autosomal recessive condition each carry one copy of a mutated gene, but they typically do not show signs and symptoms of the disorder [87].

Pathogenesis of CMD. All CMD cases with known molecular diagnosis have so far been linked to ankh nonsense mutations on chromosome 6 that underlie increased intracellular and decreased extracellular pyrophosphates $\left(\mathrm{PP}_{\mathrm{i}}\right)[82,86,88,89]$. Recent studies of $\mathrm{CMD}$ also point to the role of $\mathrm{PP}_{\mathrm{i}}$ in the regulation of the bone modeling/remodeling process. The ANKH protein is type II transmembrane with 10-12 helices, spanning the outer cell membrane, and is associated with $\mathrm{PP}_{\mathrm{i}}$ efflux (Figure 10). Most of the ankh mutations are located in cytoplasmic domains close to the C-terminus $[82,86] . \mathrm{PP}_{\mathrm{i}}$ is a major inhibitor of physiologic, pathologic tissue calcification and bone mineralization. Intracellular $\mathrm{PP}_{\mathrm{i}}$ is generated and stored largely in mitochondria, but it is also detected in endoplasmic reticulum and Golgi [90-94]. The extracellular $\mathrm{PP}_{\mathrm{i}}$ concentration in the skeletal tissue is determined by several types of cell membrane proteins: ectoenzyme PC1, which generates $\mathrm{PP}_{\mathrm{i}}$ from ATP, tissue nonspecific alkaline phosphatase (TNAP), which hydrolyzes $\mathrm{PP}_{\mathrm{i}}$ into two inorganic phosphates $\left(\mathrm{P}_{\mathrm{i}}\right)$, and $\mathrm{ANKH}$, which is involved in $\mathrm{PP}_{\mathrm{i}}$ efflux (Figure 11). While the functional role of intracellular $\mathrm{PP}_{\mathrm{i}}$ in mammalian cells remains elusive, extracellular $\mathrm{PP}_{\mathrm{i}}$ has been extensively studied for its inhibitory role in tissue calcification. Extracellular $\mathrm{PP}_{\mathrm{i}}$ directly binds to the surface 


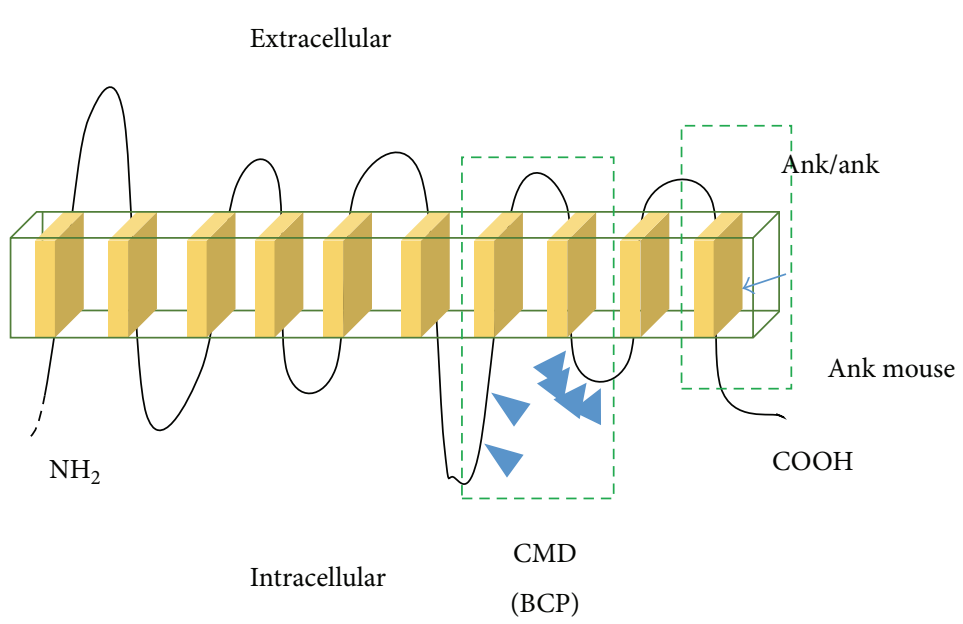

(a)

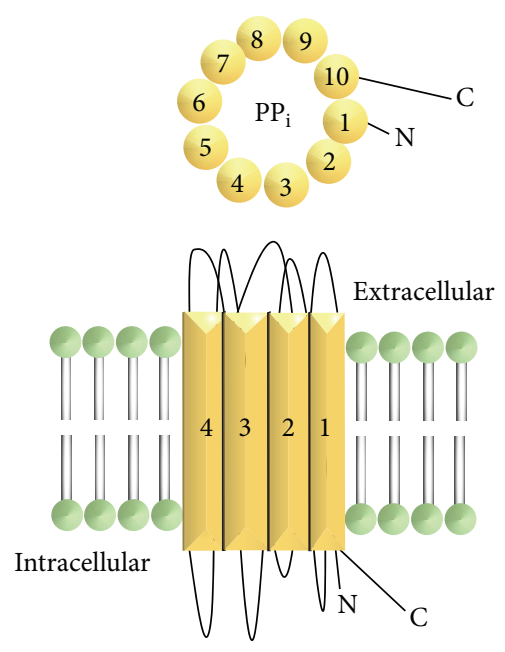

(b)

FIGURE 10: Schematic diagram of the structure of ANK protein. ANK protein is a type II transmembrane protein that spans the cell membrane with 10 helices. Most of the mutations responsible for CMD in humans fall in the intracellular sequence between 7 and 9 helix. Nonsense natural mutation in ANK mice locates toward the C-terminus on the 10th helix (a). The ANK protein works as a transporter that exports $\mathrm{PP}_{\mathrm{i}}$ from inside out of the cell (b).

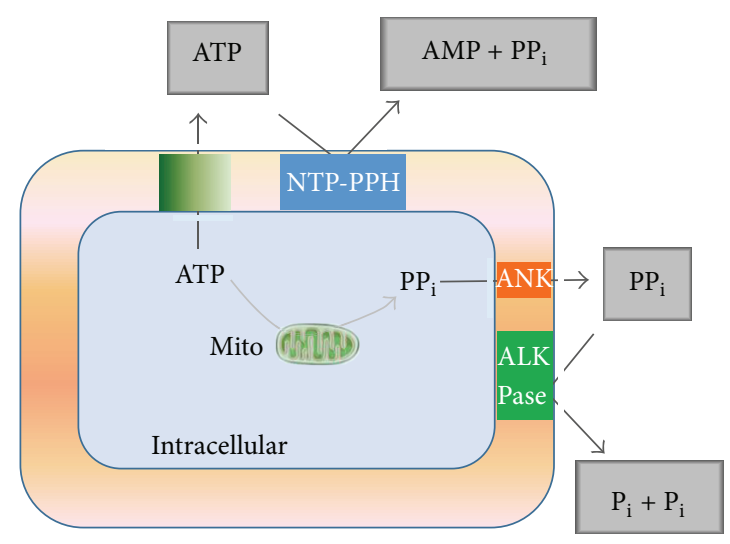

(a)

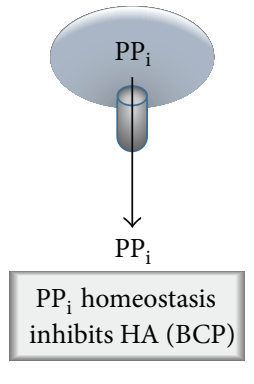

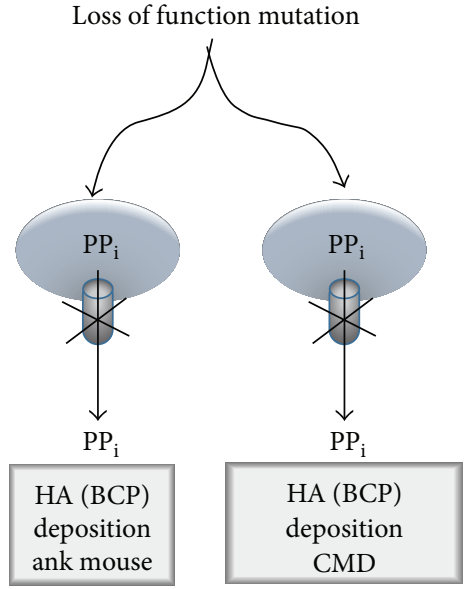

(b)

FIGURE 11: Schematic diagram of the pathogenesis of CMD. $\mathrm{PP}_{\mathrm{i}}$ is generated from ATP hydrolysis intracellular by the mitochondria (Mito) or extracellular by the transmembrane enzyme nucleoside triphosphate pyrophosphohydrolase (NTP-PPH). $\mathrm{PP}_{\mathrm{i}}$ generated intracellular is exported by ANK transporter to the extracellular one and is hydrolysed into two $P_{i}$ by alkaline phosphatase (ALP) (a). Loss of function mutation in $\mathrm{ANK}$ leads to accumulation of $\mathrm{PP}_{\mathrm{i}}$ intracellular. Absence of extracellular $\mathrm{PP}_{\mathrm{i}}$ results in excessive bone formation due to increased deposition of bone minerals; hydroxyapatite (HA) crystals made of basic calcium phosphate (BCP), responsible for CMD phenotype in humans (b).

of basic calcium phosphate hydroxyapatites and interferes with propagation of crystal formation, contributing to the formation of poorly ordered bone crystal structure $[95,96]$. In addition, exogenous $\mathrm{PP}_{\mathrm{i}}$ at micromolar concentrations stimulates the expression of osteopontin, which is a negative regulator of mineralization, and inhibits the enzymatic activity of tissue nonspecific alkaline phosphatase (ALP) in osteoblast cultures [96, 97]. Thus, a decrease in extracellular $\mathrm{PP}_{\mathrm{i}}$ may hinder normal bone remodeling, for instance, by inhibiting osteoclast differentiation or activity. In support of this notion, bone marrow-derived monocytes (BMMs) from a CMD knock-in mouse (p.Phe377del in ank) poorly differentiated to osteoclasts in cultures, compared to those from wild type mice [98]. Consistent with the mouse data, the number of bone marrow-derived osteoclast-like cells from a CMD patient was only $40 \%$ of a normal individual, and they lacked osteoclast-specific vacuolar proton pump and the ability to absorb a dentin slice [99]. The ANKH protein may have also other, unknown functions (Figure 12). 


\section{Craniometaphyseal dysplasia}

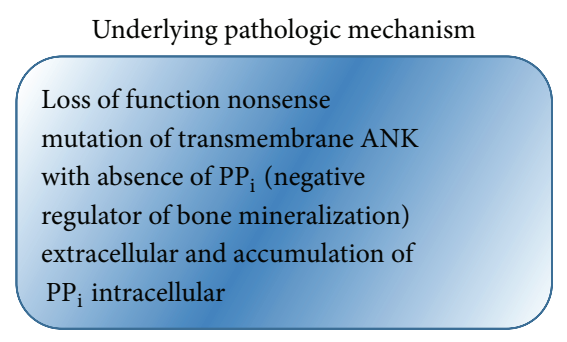

Potential therapeutic intervention

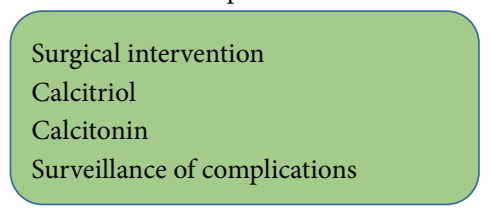

FIGURE 12: CMD pathogenesis and potential therapeutic interventions.

Treatment. Therapeutic intervention consists primarily of surgery aiming to decompress the nerve canal and/or narrowed foramen magnum. Excessive bony overgrowth of facial bone, forehead, and cranial regions can be contoured; however, bone regrowth is common. For severe complications, surgery is conservative to relieve severe symptoms caused by cranial nerve compression. Surveillance of patients is crucial as bone growth continues throughout life, and the patients will require regular neurologic evaluation, hearing assessment, and ophthalmologic examination for early diagnosis and management of complications. Therapeutic trial with calcitriol that stimulate bone resorption, with low calcium diet, has been reported to improve facial paralysis but has no effect on metaphyseal deformity [100]. Trial with calcitonin has been thought to be effective due to its inhibitory effect on bone turnover which is inefficient in treating hyperplasia of craniofacial bones in persons with CMD [101].

\section{Achondroplasia}

Achondroplasia (chondrodysplasias) is a human bone genetic disorder of the growth plate and is the most common form of dwarfism [102]. Achondroplasia is caused by AD mutations of the transmembrane receptor fibroblast growth factor receptor 3 (FGFR3), an important regulator of linear bone growth [103, 104]. Achondroplasia has an incidence rate of one in 20,000 live births; and it results from a spontaneous heterogeneous mutation to nonachondroplastic parents in an estimated $80 \%$ of cases $[102,105]$.

Clinical Diagnosis. Achondroplasia is most likely recognized at birth because of its characteristic clinical and radiographic features. Achondroplasia in newborn infants classically presents with disproportionate shortening of the limbs, a long and narrow trunk, a large head with frontal bossing, and a hypoplastic midface. The hands are short and broad, often displaying a three-pronged (trident) configuration. Moreover, many joints show hyperextensibility and infants are often hypotonic. Skeletal x-rays of the newborn infant reveal characteristic abnormalities that include shortening of the long bones of the limbs, particularly the proximal bones, with metaphyseal irregularities. The pelvis is abnormal with small and square iliac wings. The cranium is large with a prominent forehead with midface hypoplasia.
Pathogenesis. Achondroplasia is an AD genetic disorder, where it is linked to mutations of FGFR3 on the distal short arm of chromosome $4[106,107]$. Patients with achondroplasia have nonsense genetic mutation in FGFR3 with glycine to arginine substitution at position 380 (G380R), in the transmembrane domain of the receptor [105]. However, additional FGFR3 mutations have been detected in hypochondroplasia, achondroplasia with developmental delay, and acanthosis nigricans, Muenke craniosynostosis and Crouzon syndrome with acanthosis nigricans $[102,105,108]$. However, the diagnosis can be established from DNA mutational analysis. Mutational diagnosis can also be used for prenatal especially in couples at risk of having baby with homozygous achondroplasia.

FGFR3 mutations in mice have identified the function of FGFR3 in skeletal development and postnatal bone formation. The global knockout of FGFR3 generated large mice with longer than normal limb bones [109, 110]. However, knocking in FGFR3 with achondroplasia mutation in cartilage of transgenic mice produced a small mouse with short bones, a phenotype similar to those seen in human achondroplasia [111]. Collectively, these observations established the fact that FGFR3 is an important negative regulator of endochondral bone formation and that the mutations cause a constitutive activation of FGFR3, resulting in achondroplasia and related dwarfing phenotype.

Treatment. A number of therapeutic approaches have been attempted to reduce excessive activation of FGFR3 as possible treatments to normalize bone growth in achondroplasia. They include strategies to interfere with FGFR3 synthesis, block its activation, inhibit its tyrosine kinase activity, promote its degradation, and antagonize its downstream signals. These treatment modalities include FGFR3 kinase inhibitors and gamma-secretase that modulate FGFR3 cleavage and nuclear function. Another valuable therapeutic candidate in the treatment of achondroplasia is CNP that works as an antagonist to FGFR3 signal. A previous study revealed that transgenic mice overexpressing brain natriuretic peptide (BNP) in the liver exhibited postnatal skeletal overgrowth with elongation of long bone growth plates [112]. Another study showed that CNP is more potent than BNP in stimulating bone growth by using tibial organ culture experiments, suggesting that $\mathrm{CNP}$ was the physiological ligand in growing 
Achondroplasia

Underlying pathologic mechanism

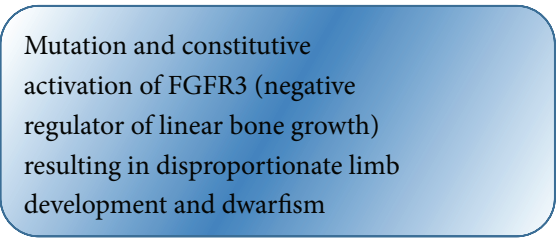

Potential therapeutic intervention

FGFR3 kinase inhibitors

Gamma secretase

CNP (FGFR3 antagonist)

FIGURE 13: Achondroplasia pathogenesis and potential therapeutic interventions.

bones [113]. Global knockout of CNP in mice showed severe postnatal dwarfism that was rescued after crossing with mice overexpressing CNP from a transgene driven by the cartilagespecific COL2A1 promoter [114]. These results confirmed the stimulatory effects of CNP on endochondral ossification in vivo. To explore the beneficial effects of CNP in treating achondroplasia, mice overexpressing CNP in cartilage were crossed with mice displaying an achondroplastic phenotype due to overexpression mutation of FGFR3 [115]. Interestingly, the skeletal growth defect in the achondroplastic mice was corrected by the local overexpression of CNP. The results suggested that CNP antagonizes the active FGFR3 possibly by inhibition of MAPK-mediated FGFR3 signaling (Figure 13).

\section{Hypophosphatasia}

Hypophosphatasia (HPP) is an inherited metabolic bone disorder [116], caused by genetic loss of function mutation(s) of tissue-nonspecific alkaline phosphatase (TNSALP) [117]. Therefore, the high extracellular inorganic pyrophosphate $\left(\mathrm{PP}_{\mathrm{i}}\right)$, a TNSALP substrate with inhibiting effects on mineralization accumulates, leads to subnormal extracellular concentrations of calcium and $\mathrm{P}_{\mathrm{i}}$ that result in rickets or osteomalacia [117]. HPP is an exception where the circulating levels are usually normal or elevated [118]. Despite the high levels of TNSALP in bone, cartilage, liver, and kidney in healthy individuals, HPP appears to disrupt only ALP in "hard tissues" directly [118]. HPP is characterized by a wideranging expressivity that ranges from death in utero with almost an unmineralized skeleton to difficulties with adult teeth without skeletal disease. Five major forms of HPP have been identified based on clinical diagnosis. The age at diagnosis of skeletal disease determines the perinatal, infantile, childhood, and adult types of HPP [118]. Individuals without skeletal findings but dental features only are said to have "odonto-HPP" [118]. Autosomal recessive (AR) and autosomal dominant (AD) inheritance partially explain the remarkable range of HPP severity [117]. Perinatal and infantile HPP cases are inherited as an AR trait, whereas the more mild forms may reflect $\mathrm{AR}$ or $\mathrm{AD}$ inheritance $[117,119]$. To date, 224 different defects in TNSALP $(80 \%$ missense mutation) have been identified in HPP that explain the extreme range of severity of this disorder. The prognoses for these five major forms of HPP are determined by the skeletal complications. Typically, the earlier the signs and symptoms, the worse the outcome [118].
Pathogenesis of HPP. The bone disease is due to missense mutation of TNSALP with structural defects. Many TNSALP mutations responsible for HPP change a conserved amino acid in the mammalian TNSALPs [120]. Some mutations disturb the catalytic pocket or the structural binding site for metal ligand; others compromise dimer formation [118, 120]. Moreover, some mutations impair the intracellular movement of TNSALP [120]. TNSALP deficient mice have confirmed insight from HPP patients and showed reduced longitudinal growth and delayed epiphyseal ossification, accompanied by disturbance in the mineralization pattern. It is concluded that ablation of TNALP results in hypomineralization of the skeleton with sever disordered mineralized matrix architecture [121].

Prognosis. Perinatal HPP is always fatal. Infantile HPP often features clinical and radiographic deterioration with approximately $50 \%$ of babies dying from respiratory compromise $[122,123]$. Childhood HPP may get improved after fusion of the growth plates. Skeletal problems are likely to return in adulthood [124]. Adult HPP causes recurrent and long lasting orthopedic difficulties (Figure 14).

Treatment. There is no established therapeutic protocol of HPP, although several approaches have been attempted, including intravenous infusions of soluble recombinant ALP [125], bone marrow transplantation [123], and teriparatide administration [124]. Bisphosphonates (derivatives of $\mathrm{PP}_{\mathrm{i}}$ ) could be ineffective or pose further problems [118]. It has been reported that plasma and urine $\mathrm{PP}_{\mathrm{i}}$ decrease after placental ALP correction of the hypophosphatasia in pregnant carriers of HPP [118] and i.v. injection of purified placental ALP was used to correct hypophosphatasemia in a severely affected infant, but there was no clinical or radiographic improvement. These negative results suggested the greater tissue need for ALP, or perhaps ALP must be bound to plasma membranes for therapeutic efficacy.

\section{Conclusion}

There is yet a large scale of work needed to be done towards the discovery of new therapeutic methods of rare genetic bone disorders. The elucidation of disease mechanisms will provide the first step. Several potential therapeutic interventions have been proposed; however, implementation of these therapeutic strategies will take time. The disease 
Hypophosphatasia

Underlying pathologic mechanism

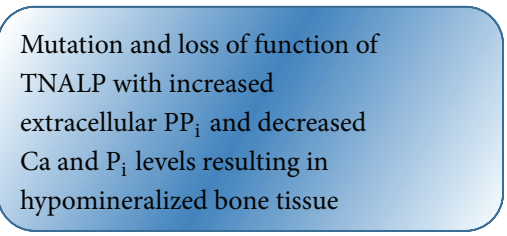

Potential therapeutic intervention

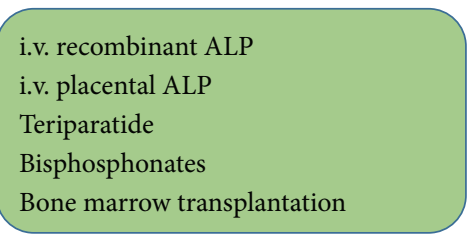

FIGURE 14: Hypophosphatasia pathogenesis and potential therapeutic interventions.

mechanism of Gorham-Stout disease, melorheostosis, and multiple hereditary exostosis still needs to be fully elucidated. The development of inhibitors of the ACVR1/ALK2 pathway seems to show promise as a possible therapeutic intervention for FOP. The use of bisphosphonates and IL- 6 inhibitors may be useful in the treatment of fibrous dysplasia, but further studies are needed. A viable cell therapy, bisphosphonate polytherapy, and HGH may have potential to avert the pathology in osteogenesis imperfecta, but more research is needed to prove therapeutic benefit.

The need for cures to these rare bone disorders has never been more pressing, given the increasing number of afflicted individuals living across the globe. Furthermore, potential cures for these rare bone disorders may also impact the management of more common bone diseases that display the same basic mechanisms such as heterotrophic ossification. Thus, research in the upcoming years will show that viable therapies of rare bone disorders might be in the horizons.

\section{Conflict of Interests}

The authors declare that there is no conflict of interests.

\section{References}

[1] R. Rubin, D. S. Strayer, and E. Rubin, Rubin's Pathology: Clinicopathologic Foundations of Medicine, Wolters Kluwer Health/Lippincott Williams \& Wilkins, Philadelphia, Pa, USA, 6th edition, 2012.

[2] B. W. Neville, Oral and Maxillofacial Pathology, Saunders/Elsevier, St. Louis, Mo, USA, 3rd edition, 2009.

[3] S. Yetiser, E. Gonul, F. Tosun, M. Tasar, and Y. Hidir, "Monostotic craniofacial fibrous dysplasia: the Turkish experience," Journal of Craniofacial Surgery, vol. 17, no. 1, pp. 62-67, 2006.

[4] R. D. Chapurlat and P. Orcel, "Fibrous dysplasia of bone and McCune-Albright syndrome," Best Practice \& Research: Clinical Rheumatology, vol. 22, no. 1, pp. 55-69, 2008.

[5] J. B. Regard, N. Cherman, D. Palmer et al., "Wnt/ $\beta$-catenin signaling is differentially regulated by $\mathrm{G} \alpha$ proteins and contributes to fibrous dysplasia," Proceedings of the National Academy of Sciences of the United States of America, vol. 108, no. 50, pp. 20101-20106, 2011.

[6] T. G. Kashima, T. Nishiyama, K. Shimazu et al., "Periostin, a novel marker of intramembranous ossification, is expressed in fibrous dysplasia and in c-Fos-overexpressing bone lesions," Human Pathology, vol. 40, no. 2, pp. 226-237, 2009.
[7] I. Saggio, C. Remoli, E. Spica et al., "Constitutive expression of $\mathrm{G} s \alpha^{\mathrm{R} 201 \mathrm{C}}$ in mice produces a heritable, direct replica of human fibrous dysplasia bone pathology and demonstrates its natural history," Journal of Bone and Mineral Research, 2014.

[8] M. G. Su, R. Tian, Q. P. Fan et al., "Recognition of fibrous dysplasia of bone mimicking skeletal metastasis on 18F-FDG PET/CT imaging," Skeletal Radiology, vol. 40, no. 3, pp. 295302, 2011.

[9] F. Tabareau-Delalande, C. Collin, A. Gomez-Brouchet et al., "Diagnostic value of investigating GNAS mutations in fibroosseous lesions: a retrospective study of 91 cases of fibrous dysplasia and 40 other fibro-osseous lesions," Modern Pathology, vol. 26, no. 7, pp. 911-921, 2013.

[10] A. A. Mäkitie, J. Törnwall, and O. Mäkitie, "Bisphosphonate treatment in craniofacial fibrous dysplasia-a case report and review of the literature," Clinical Rheumatology, vol. 27, no. 6, pp. 809-812, 2008.

[11] R. D. Chapurlat, D. Gensburger, J. M. Jimenez-Andrade, J. R. Ghilardi, M. Kelly, and P. Mantyh, "Pathophysiology and medical treatment of pain in fibrous dysplasia of bone," Orphanet Journal of Rare Diseases, vol. 7, no. 1, article S3, 2012.

[12] P. Garnero, E. Thompson, T. Woodworth, and J. S. Smolen, "Rapid and sustained improvement in bone and cartilage turnover markers with the anti-interleukin- 6 receptor inhibitor tocilizumab plus methotrexate in rheumatoid arthritis patients with an inadequate response to methotrexate: results from a substudy of the multicenter double-blind, placebo-controlled trial of tocilizumab in inadequate responders to methotrexate alone," Arthritis and Rheumatism, vol. 62, no. 1, pp. 33-43, 2010.

[13] D. P. Minde, Z. Anvarian, S. G. D. Rüdiger, and M. M. Maurice, "Messing up disorder: how do missense mutations in the tumor suppressor protein APC lead to cancer?" Molecular Cancer, vol. 10, article 101, 2011.

[14] K. Radhakrishnan and S. G. Rockson, "Gorham's disease: an osseous disease of lymphangiogenesis?" Annals of the New York Academy of Sciences, vol. 1131, pp. 203-205, 2008.

[15] W. M. Tsang, A. C. Tong, L. T. Chow, and I. O. Ng, "Massive osteolysis (Gorham disease) of the maxillofacial skeleton: report of 2 cases," Journal of Oral and Maxillofacial Surgery, vol. 62, no. 2, pp. 225-230, 2004.

[16] T. Hirayama, A. Sabokbar, I. Itonaga, S. Watt-Smith, and N. A. Athanasou, "Cellular and humoral mechanisms of osteoclast formation and bone resorption in Gorham-Stout disease," The Journal of Pathology, vol. 195, no. 5, pp. 624-630, 2001.

[17] R. Venkatramani, N. S. Ma, P. Pitukcheewanont, M. H. Malogolowkin, and L. Mascarenhas, "Gorham's disease and diffuse lymphangiomatosis in children and adolescents," Pediatric Blood and Cancer, vol. 56, no. 4, pp. 667-670, 2011. 
[18] D. Bruch-Gerharz, C.-D. Gerharz, H. Stege et al., "Cutaneous lymphatic malformations in disappearing bone (GorhamStout) disease: a novel clue to the pathogenesis of a rare syndrome," Journal of the American Academy of Dermatology, vol. 56, supplement 2, pp. S21-S25, 2007.

[19] S. Ray, S. Mukhopadhyay, R. Bandyopadhyay, and S. K. Sinha, "Vanishing bone disease (Gorham' disease) - a rare occurrence of unknown etiology," Indian Journal of Pathology and Microbiology, vol. 55, no. 3, pp. 399-401, 2012.

[20] M. T. Dellinger, N. Garg, and B. R. Olsen, "Viewpoints on vessels and vanishing bones in Gorham-Stout disease," Bone, vol. 63, pp. 47-52, 2014.

[21] T. Mukai, F. Otsuka, H. Otani et al., "TNF- $\alpha$ inhibits BMP-induced osteoblast differentiation through activating SAPK/JNK signaling," Biochemical and Biophysical Research Communications, vol. 356, no. 4, pp. 1004-1010, 2007.

[22] R. D. Devlin, H. G. Bone III, and G. D. Roodman, "Interleukin6: a potential mediator of the massive osteolysis in patients with Gorham-Stout disease," The Journal of Clinical Endocrinology and Metabolism, vol. 81, no. 5, pp. 1893-1897, 1996.

[23] J. Fontanesi, "Radiation therapy in the treatment of Gorham disease," Journal of Pediatric Hematology/Oncology, vol. 25, no. 10, pp. 816-817, 2003.

[24] F. Hammer, W. Kenn, U. Wesselmann et al., "Gorham-Stout disease-stabilization during bisphosphonate treatment," Journal of Bone and Mineral Research, vol. 20, no. 2, pp. 350-353, 2005.

[25] A. Franchi, F. Bertoni, P. Bacchini, V. Mourmouras, and C. Miracco, "CD105/endoglin expression in Gorham disease of bone," Journal of Clinical Pathology, vol. 62, no. 2, pp. 163-167, 2009.

[26] F. S. Kaplan, M. Le Merrer, D. L. Glaser et al., "Fibrodysplasia ossificans progressiva," Best Practice and Research: Clinical Rheumatology, vol. 22, no. 1, pp. 191-205, 2008.

[27] J. M. Connor and D. A. P. Evans, "Genetic aspects of fibrodysplasia ossificans progressiva," Journal of Medical Genetics, vol. 19, no. 1, pp. 35-39, 1982.

[28] L. S. de La Peña, P. C. Billings, J. L. Fiori, J. Ahn, F. S. Kaplan, and E. M. Shore, "Fibrodysplasia ossificans progressiva (FOP), a disorder of ectopic osteogenesis, misregulates cell surface expression and trafficking of BMPRIA," Journal of Bone and Mineral Research, vol. 20, no. 7, pp. 1168-1176, 2005.

[29] D. M. Rocke, M. Zasloff, J. Peeper, R. B. Cohen, and F. S. Kaplan, "Age- and joint-specific risk of initial heterotopic ossification in patients who have fibrodysplasia ossificans progressiva," Clinical Orthopaedics and Related Research, no. 301, pp. 243-248, 1994.

[30] F. S. Kaplan, M. A. Zasloff, J. A. Kitterman, E. M. Shore, C. C. Hong, and D. M. Rocke, "Early mortality and cardiorespiratory failure in patients with fibrodysplasia ossificans progressiva," The Journal of Bone and Joint Surgery American Volume A, vol. 92, no. 3, pp. 686-691, 2010.

[31] J. A. Kitterman, S. Kantanie, D. M. Rocke, and F. S. Kaplan, "Iatrogenic harm caused by diagnostic errors in fibrodysplasia ossificans progressiva," Pediatrics, vol. 116, no. 5, pp. e654-e661, 2005.

[32] S. A. Chakkalakal, D. Zhang, A. L. Culbert et al., "An Acvr1 R206H knock-in mouse has fibrodysplasia ossificans progressiva," Journal of Bone and Mineral Research, vol. 27, no. 8, pp. 1746-1756, 2012.

[33] L. Mao, M. Yano, N. Kawao, Y. Tamura, K. Okada, and H. Kaji, "Role of matrix metalloproteinase-10 in the BMP-2 inducing osteoblastic differentiation," Endocrine Journal, vol. 60, no. 12, pp. 1309-1319, 2013.

[34] F. Giacopelli, S. Cappato, L. Tonachini et al., "Identification and characterization of regulatory elements in the promoter of ACVR1, the gene mutated in Fibrodysplasia Ossificans Progressiva," Orphanet Journal of Rare Diseases, vol. 8, no. 1, article 145, 2013.

[35] M. Mura, S. Cappato, F. Giacopelli, R. Ravazzolo, and R. Bocciardi, "The role of the 3'UTR region in the regulation of the acvr1/alk-2 gene expression," PLoS ONE, vol. 7, no. 12, Article ID e50958, 2012.

[36] H. Song, Q. Wang, J. Wen et al., "ACVR1, a therapeutic target of fibrodysplasia ossificans progressiva, is negatively regulated by miR-148a," International Journal of Molecular Sciences, vol. 13, no. 2, pp. 2063-2077, 2012.

[37] S. Shi, J. Cai, D. J. de Gorter, and et al, "Antisenseoligonucleotide mediated exon skipping in activin-receptorlike kinase 2: inhibiting the receptor that is overactive in fibrodysplasia ossificans progressiva," PLoS ONE, vol. 8, no. 7, Article ID e69096, 2013.

[38] J. Kaplan, F. S. Kaplan, and E. M. Shore, "Restoration of normal BMP signaling levels and osteogenic differentiation in FOP mesenchymal progenitor cells by mutant allele-specific targeting," Gene Therapy, vol. 19, no. 7, pp. 786-790, 2012.

[39] A. M. Motimaya and S. P. Meyers, "Melorheostosis involving the cervical and upper thoracic spine: radiographic, CT, and MR imaging findings," The American Journal of Neuroradiology, vol. 27, no. 6, pp. 1198-1200, 2006.

[40] A. M. Judkiewicz, M. D. Murphey, C. S. Resnik, A. H. Newberg, H. T. Temple, and W. S. Smith, "Advanced imaging of melorheostosis with emphasis on MRI," Skeletal Radiology, vol. 30, no. 8, pp. 447-453, 2001.

[41] S. C. Zeiller, A. R. Vaccaro, D. W. Wimberley, T. J. Albert, J. S. Harrop, and A. S. Hilibrand, "Severe myelopathy resulting from melorheostosis of the cervicothoracic spine. A case report," Journal of Bone and Joint Surgery-Series A, vol. 87, no. 12 I, pp. 2759-2762, 2005.

[42] N. T. Kalbermatten, P. Vock, D. Rüfenacht, and S. E. Anderson, "Progressive melorheostasis in the peripheral and axial skeleton with associated vascular malformations: imaging findings over three decades," Skeletal Radiology, vol. 30, no. 1, pp. 48-52, 2001.

[43] M. McCarthy, H. Mehdian, K. J. Fairbairn, and A. Stevens, "Melorheostosis of the tenth and eleventh thoracic vertebrae crossing the facet joint: a rare cause of back pain," Skeletal Radiology, vol. 33, no. 5, pp. 283-286, 2004.

[44] P. A. Robertson, A. S. Don, and M. V. Miller, "Painful lumbosacral melorheostosis treated by fusion," Spine, vol. 28, no. 12, pp. E234-E238, 2003.

[45] R. J. Hollick, A. Black, and D. Reid, "Melorheostosis and its treatment with intravenous zoledronic acid," BMJ Case Reports, 2010.

[46] E. Moulder and C. Marsh, "Soft tissue knee contracture of the knee due to melorheostosis, treated by total knee arthroplasty," The Knee, vol. 13, no. 5, pp. 395-396, 2006.

[47] J. Hellemans, O. Preobrazhenska, A. Willaert et al., "Lossof-function mutations in LEMD3 result in osteopoikilosis, Buschke-Ollendorff syndrome and melorheostosis," Nature Genetics, vol. 36, no. 11, pp. 1213-1218, 2004.

[48] J. Hellemans, P. Debeer, M. Wright et al., "Germline LEMD3 mutations are rare in sporadic patients with isolated melorheostosis," Human mutation., vol. 27, no. 3, p. 290, 2006. 
[49] J. E. Kim, E. H. Kim, E. H. Han et al., "A TGF-betainducible cell adhesion molecule, betaig-h3, is downregulated in melorheostosis and involved in osteogenesis," Journal of Cellular Biochemistry, vol. 77, no. 2, pp. 169-178, 2000.

[50] H. Endo, A. Katsumi, K. Kuroda, A. Utani, H. Moriya, and H. Shinkai, "Increased procollagen $\alpha 1$ (I) mRNA expression by dermal fibroblasts in melorheostosis," The British Journal of Dermatology, vol. 148, no. 4, pp. 799-803, 2003.

[51] A. Kivioja, H. Ervasti, J. Kinnunen, I. Kaitila, M. Wolf, and T. Böhling, "Chondrosarcoma in a family with multiple hereditary exostoses," Journal of Bone and Joint Surgery-Series B, vol. 82, no. 2, pp. 261-266, 2000.

[52] G. A. Schmale, E. U. Conrad III, and W. H. Raskind, "The natural history of hereditary multiple exostoses," The Journal of Bone and Joint Surgery-Series A, vol. 76, no. 7, pp. 986-992, 1994.

[53] W. Wuyts and W. Van Hul, "Molecular basis of multiple exostoses: mutations in the EXT1 and EXT2 genes," Human Mutation, vol. 15, no. 3, pp. 220-227, 2000.

[54] M. Busse, A. Feta, J. Presto et al., "Contribution of EXT1, EXT2, and EXTL3 to heparan sulfate chain elongation," Journal of Biological Chemistry, vol. 282, no. 45, pp. 32802-32810, 2007.

[55] C. Francannet, A. Cohen-Tanugi, M. Le Merrer, A. Munnich, J. Bonaventure, and L. Legeai-Mallet, "Genotype-phenotype correlation in hereditary multiple exostoses," Journal of Medical Genetics, vol. 38, no. 7, pp. 430-434, 2001.

[56] D. E. Porter, L. Lonie, M. Fraser et al., "Severity of disease and risk of malignant change in hereditary multiple exostoses," Journal of Bone and Joint Surgery-Series B, vol. 86, no. 7, pp. 1041-1046, 2004.

[57] K. Matsumoto, F. Irie, S. Mackem, and Y. Yamaguchi, "A mouse model of chondrocyte-specific somatic mutation reveals a role for Ext1 loss of heterozygosity in multiple hereditary exostoses," Proceedings of the National Academy of Sciences of the United States of America, vol. 107, no. 24, pp. 10932-10937, 2010.

[58] Y.-E. Shieh, D. E. Wells, and A. K. Sater, "Zygotic expression of exostosin1 (Ext1) is required for BMP signaling and establishment of dorsal-ventral pattern in Xenopus," The International Journal of Developmental Biology, vol. 58, no. 1, pp. 27-34, 2014.

[59] L. Cantley, C. Saunders, M. Guttenberg et al., "Loss of $\beta$-catenin induces multifocal periosteal chondroma-like masses in mice," The American Journal of Pathology, vol. 182, no. 3, pp. 917-927, 2013.

[60] M. F. Anower-E-Khuda, K. Matsumoto, H. Habuchi et al., "Glycosaminoglycans in the blood of hereditary multiple exostoses patients: Half reduction of heparan sulfate to chondroitin sulfate ratio and the possible diagnostic application," Glycobiology, vol. 23, no. 7, pp. 865-876, 2013.

[61] J. R. Stieber and J. P. Dormans, "Manifestations of hereditary multiple exostoses.", The Journal of the American Academy of Orthopaedic Surgeons, vol. 13, no. 2, pp. 110-120, 2005.

[62] K. B. Jones, "Glycobiology and the growth plate: current concepts in multiple hereditary exostoses," Journal of Pediatric Orthopaedics, vol. 31, no. 5, pp. 577-586, 2011.

[63] S. H. Kozin, "Congenital differences about the elbow," Hand Clinics, vol. 25, no. 2, pp. 277-291, 2009.

[64] I. Solomon, "Chondrosarcoma in hereditary multiple exostosis," South African Medical Journal, vol. 48, no. 16, pp. 671-676, 1974.

[65] R. C. M. Hennekam, "Hereditary multiple exostoses," Journal of Medical Genetics, vol. 28, no. 4, pp. 262-266, 1991.
[66] J. V. M. G. Bovée, "Multiple osteochondromas," Orphanet Journal of Rare Diseases, vol. 3, no. 1, article 3, 2008.

[67] I. Khan, C. A. West Jr., G. P. Sangster, M. Heldmann, L. Doucet, and M. Olmedo, "Multiple hereditary exostoses as a rare nonatherosclerotic etiology of chronic lower extremity ischemia," Journal of Vascular Surgery, vol. 51, no. 4, pp. 10031005, 2010.

[68] R. D. Steiner, J. Adsit, and D. Basel, "COL1A1/2-related osteogenesis imperfecta," in GeneReviews, R. A. Pagon, M. P. Adam, H. H. Ardinger et al., Eds., 1993.

[69] J. C. Marini, W. A. Cabral, A. M. Barnes, and W. Chang, "Components of the collagen prolyl 3-hydroxylation complex are crucial for normal bone development," Cell Cycle, vol. 6, no. 14, pp. 1675-1681, 2007.

[70] T. E. Uveges, P. Collin-Osdoby, W. A. Cabral et al., "Cellular mechanism of decreased bone in Brtl mouse model of OI: imbalance of decreased osteoblast function and increased osteoclasts and their precursors," Journal of Bone and Mineral Research, vol. 23, no. 12, pp. 1983-1994, 2008.

[71] R. Bargman, R. Posham, A. L. Boskey, E. Dicarlo, C. Raggio, and N. Pleshko, "Comparable outcomes in fracture reduction and bone properties with RANKL inhibition and alendronate treatment in a mouse model of osteogenesis imperfecta," Osteoporosis International, vol. 23, no. 3, pp. 1141-1150, 2012.

[72] F. S. van Dijk, J. M. Cobben, A. Kariminejad et al., "Osteogenesis imperfecta: a review with clinical examples," Molecular Syndromology, vol. 2, no. 1, pp. 1-20, 2011.

[73] J. C. Marini and N. L. Gerber, "Osteogenesis imperfecta: rehabilitation and prospects for gene therapy," The Journal of the American Medical Association, vol. 277, no. 9, pp. 746-750, 1997.

[74] C. A. Phillipi, T. Remmington, and R. D. Steiner, "Bisphosphonate therapy for osteogenesis imperfecta," Cochrane Database of Systematic Reviews, no. 4, Article ID CD005088, 2008.

[75] R. Sakkers, D. Kok, R. Engelbert et al., "Skeletal effects and functional outcome with olpadronate in children with osteogenesis imperfecta: a 2-year randomised placebo-controlled study," The Lancet, vol. 363, no. 9419, pp. 1427-1431, 2004.

[76] F. Antoniazzi, E. Monti, G. Venturi et al., "GH in combination with bisphosphonate treatment in osteogenesis imperfecta," European Journal of Endocrinology, vol. 163, no. 3, pp. 479-487, 2010.

[77] J. C. Marini, E. Hopkins, F. H. Glorieux et al., "Positive linear growth and bone responses to growth hormone treatment in children with types III and IV osteogenesis imperfecta: high predictive value of the carboxyterminal propeptide of type I procollagen," Journal of Bone and Mineral Research, vol. 18, no. 2, pp. 237-243, 2003.

[78] S. Otsuru, P. L. Gordon, K. Shimono et al., "Transplanted bone marrow mononuclear cells and MSCs impart clinical benefit to children with osteogenesis imperfecta through different mechanisms," Blood, vol. 120, no. 9, pp. 1933-1941, 2012.

[79] D. B. Kirkpatrick, "Craniometaphyseal dysplasia," Surgical Neurology, vol. 28, no. 3, p. 231, 1987.

[80] D. E. Cole and M. M. Cohen Jr., "A new look at craniometaphyseal dysplasia," Journal of Pediatrics, vol. 112, no. 4, pp. 577-578, 1988.

[81] P. Beighton, "Craniometaphyseal dysplasia (CMD), autosomal dominant form," Journal of Medical Genetics, vol. 32, no. 5, pp. 370-374, 1995.

[82] P. Nürnberg, H. Thiele, D. Chandler et al., "Heterozygous mutations in $\mathrm{ANKH}$, the human ortholog of the mouse progressive 
ankylosis gene, result in craniometaphyseal dysplasia," Nature Genetics, vol. 28, no. 1, pp. 37-41, 2001.

[83] T. Kato, H. Matsumoto, A. Chida, H. Wakamatsu, and S. Nonoyama, "Maternal mosaicism of an ANKH mutation in a family with craniometaphyseal dysplasia," Pediatrics International, vol. 55, no. 2, pp. 254-256, 2013.

[84] G. Baynam, J. Goldblatt, and L. Schofield, "Craniometaphyseal dysplasia and chondrocalcinosis cosegregating in a family with an ANKH mutation," The American Journal of Medical Genetics, Part A, vol. 149, no. 6, pp. 1331-1333, 2009.

[85] S. Tinschert and H. S. Braun, "Craniometaphyseal dysplasia in six generations of a German kindred," American Journal of Medical Genetics, vol. 77, no. 3, pp. 175-181, 1998.

[86] E. Reichenberger, V. Tiziani, S. Watanabe et al., "Autosomal dominant craniometaphyseal dysplasia is caused by mutations in the transmembrane protein ANK," The American Journal of Human Genetics, vol. 68, no. 6, pp. 1321-1326, 2001.

[87] Y. Hu, I.-P. Chen, S. de Almeida et al., "A novel autosomal recessive GJA1 missense mutation linked to Craniometaphyseal dysplasia," PLoS ONE, vol. 8, no. 8, Article ID e73576, 2013.

[88] K. A. Gurley, R. J. Reimer, and D. M. Kingsley, "Biochemical and genetic analysis of ANK in arthritis and bone disease," American Journal of Human Genetics, vol. 79, no. 6, pp. 1017-1029, 2006.

[89] A. M. Ho, M. D. Johnson, and D. M. Kingsley, "Role of the mouse ank gene in control of tissue calcification and arthritis," Science, vol. 289, no. 5477, pp. 265-270, 2000.

[90] S. E. Mansurova, "Inorganic pyrophosphate in mitochondrial metabolism," Biochimica et Biophysica Acta, vol. 977, no. 3, pp. 237-247, 1989.

[91] A. M. Davidson and A. P. Halestrap, "Inorganic pyrophosphate is located primarily in the mitochondria of the hepatocyte and increases in parallel with the decrease in light-scattering induced by gluconeogenic hormones, butyrate and ionophore A23187," Biochemical Journal, vol. 254, no. 2, pp. 379-384, 1988.

[92] J. W. Rachow and L. M. Ryan, "Inorganic pyrophosphate metabolism in arthritis," Rheumatic Disease Clinics of North America, vol. 14, no. 2, pp. 289-302, 1988.

[93] J. M. Capasso, T. W. Keenan, C. Abeijon, and C. B. Hirschberg, "Mechanism of phosphorylation in the lumen of the Golgi apparatus. Translocation of adenosine 5 '-triphosphate into Golgi vesicles from rat liver and mammary gland," Journal of Biological Chemistry, vol. 264, no. 9, pp. 5233-5240, 1989.

[94] K. Johnson, A. Jung, A. Murphy, A. Andreyev, J. Dykens, and R. Terkeltaub, "Mitochondrial oxidative phosphorylation is a downstream regulator of nitric oxide effects on chondrocyte matrix synthesis and mineralization," Arthritis \& Rheumatology, vol. 43, no. 7, pp. 1560-1570, 2000.

[95] H. Fleisch, R. G. G. Russell, and F. Straumann, "Effect of pyrophosphate on hydroxyapatite and its implications in calcium homeostasis," Nature, vol. 212, no. 5065, pp. 901-903, 1966.

[96] W. N. Addison, F. Azari, E. S. Sørensen, M. T. Kaartinen, and M. D. McKee, "Pyrophosphate inhibits mineralization of osteoblast cultures by binding to mineral, up-regulating osteopontin, and inhibiting alkaline phosphatase activity," Journal of Biological Chemistry, vol. 282, no. 21, pp. 15872-15883, 2007.

[97] D. Harmey, L. Hessle, S. Narisawa, K. A. Johnson, R. Terkeltaub, and J. L. Millán, "Concerted regulation of inorganic pyrophosphate and osteopontin by akp2, enpp1, and ank: an integrated model of the pathogenesis of mineralization disorders," The American Journal of Pathology, vol. 164, no. 4, pp. 1199-1209, 2004.
[98] I.-P. Chen, C. J. Wang, S. Strecker, B. Koczon-Jaremko, A. Boskey, and E. J. Reichenberger, "Introduction of a Phe377del mutation in ANK creates a mouse model for craniometaphyseal dysplasia," Journal of Bone and Mineral Research, vol. 24, no. 7, pp. 1206-1215, 2009.

[99] T. Yamamoto, N. Kurihara, K. Yamaoka et al., "Bone marrowderived osteoclast-like cells from a patient with craniometaphyseal dysplasia lack expression of osteoclast-reactive vacuolar proton pump," Journal of Clinical Investigation, vol. 91, no. 1, pp. 362-367, 1993.

[100] L. Lyndon Key Jr., F. Volberg, R. Baron, and C. S. Anast, "Treatment of craniometaphyseal dysplasia with calcitriol," The Journal of Pediatrics, vol. 112, no. 4, pp. 583-587, 1988.

[101] S. Fanconi, J. A. Fischer, P. Wieland et al., "Craniometaphyseal dysplasia with increased bone turnover and secondary hyperparathyroidism: therapeutic effect of calcitonin," Journal of Pediatrics, vol. 112, no. 4, pp. 587-591, 1988.

[102] W. A. Horton, J. G. Hall, and J. T. Hecht, "Achondroplasia," The Lancet, vol. 370, no. 9582, pp. 162-172, 2007.

[103] R. Shiang, L. M. Thompson, Y.-Z. Zhu et al., "Mutations in the transmembrane domain of FGFR3 cause the most common genetic form of dwarfism, achondroplasia," Cell, vol. 78, no. 2, pp. 335-342, 1994.

[104] G. A. Bellus, T. W. Hefferon, R. I. de Ortiz Luna et al., "Achondroplasia is defined by recurrent G380R mutations of FGFR3," The American Journal of Human Genetics, vol. 56, no. 2, pp. 367-373, 1995.

[105] G. A. Bellus, I. McIntosh, E. A. Smith et al., "A recurrent mutation in the tyrosine kinase domain of fibroblast growth factor receptor 3 causes hypochondroplasia," Nature Genetics, vol. 10, no. 3, pp. 357-359, 1995.

[106] F. Rousseau, J. Bonaventure, L. Legeai-Mallet et al., "Mutations in the gene encoding fibroblast growth factor receptor-3 in achondroplasia," Nature, vol. 371, no. 6494, pp. 252-254, 1994.

[107] M. Velinov, S. A. Slaugenhaupt, I. Stoilov, C. I. Scott Jr., J. F. Gusella, and P. Tsipouras, "The gene for achondroplasia maps to the telomeric region of chromosome 4p," Nature Genetics, vol. 6, no. 3, pp. 314-317, 1994.

[108] Z. Vajo, C. A. Francomano, and D. J. Wilkin, “The molecular and genetic basis of fibroblast growth factor receptor 3 disorders: the achondroplasia family of skeletal dysplasias, Muenke craniosynostosis, and Crouzon syndrome with acanthosis nigricans," Endocrine Reviews, vol. 21, no. 1, pp. 23-39, 2000.

[109] J. S. Colvin, B. A. Bohne, G. W. Harding, D. G. McEwen, and D. M. Ornitz, "Skeletal overgrowth and deafness in mice lacking fibroblast growth factor receptor 3," Nature Genetics, vol. 12, no. 4, pp. 390-397, 1996.

[110] C. Deng, A. Wynshaw-Boris, F. Zhou, A. Kuo, and P. Leder, "Fibroblast growth factor receptor 3 is a negative regulator of bone growth," Cell, vol. 84, no. 6, pp. 911-921, 1996.

[111] M. C. Naski, J. S. Colvin, J. Douglas Coffin, and D. M. Ornitz, "Repression of hedgehog signaling and BMP4 expression in growth plate cartilage by fibroblast growth factor receptor 3," Development, vol. 125, no. 24, pp. 4977-4988, 1998.

[112] M. Suda, Y. Ogawa, K. Tanaka et al., "Skeletal overgrowth in transgenic mice that overexpress brain natriuretic peptide," Proceedings of the National Academy of Sciences of the United States of America, vol. 95, no. 5, pp. 2337-2342, 1998.

[113] A. Yasoda, Y. Ogawa, M. Suda et al., "Natriuretic peptide regulation of endochondral ossification: Evidence for possible roles of the C-type natriuretic peptide/guanylyl cyclase-B pathway," 
Journal of Biological Chemistry, vol. 273, no. 19, pp. 11695-11700, 1998.

[114] H. Chusho, N. Tamura, Y. Ogawa et al., "Dwarfism and early death in mice lacking C-type natriuretic peptide," Proceedings of the National Academy of Sciences of the United States of America, vol. 98, no. 7, pp. 4016-4021, 2001.

[115] A. Yasoda, Y. Komatsu, H. Chusho et al., "Overexpression of CNP in chondrocytes rescues achondroplasia through a MAPK-dependent pathway," Nature Medicine, vol. 10, no. 1, pp. 80-86, 2004.

[116] P. S. Henthorn, M. Raducha, K. N. Fedde, M. A. Lafferty, and M. P. Whyte, "Different missense mutations at the tissuenonspecific alkaline phosphatase gene locus in autosomal recessively inherited forms of mild and severe hypophosphatasia," Proceedings of the National Academy of Sciences of the United States of America, vol. 89, no. 20, pp. 9924-9928, 1992.

[117] D. P. Ramadža, F. Stipoljev, V. Sarnavka et al., "Hypophosphatasia: phenotypic variability and possible Croatian origin of the c.1402G>A mutation of TNSALP gene," Collegium Antropologicum, vol. 33, no. 4, pp. 1255-1258, 2009.

[118] M. P. Whyte, M. Landt, L. M. Ryan et al., "Alkaline phosphatase: placental and tissue-nonspecific isoenzymes hydrolyze phosphoethanolamine, inorganic pyrophosphate, and pyridoxal $5^{\prime}$ phosphate. Substrate accumulation in carriers of hypophosphatasia corrects during pregnancy," The Journal of Clinical Investigation, vol. 95, no. 4, pp. 1440-1445, 1995.

[119] S. Mumm, J. Jones, P. Finnegan, P. S. Henthorn, M. N. Podgornik, and M. P. Whyte, "Denaturing gradient gel electrophoresis analysis of the tissue nonspecific alkaline phosphatase isoenzyme gene in hypophosphatasia," Molecular Genetics and Metabolism, vol. 75, no. 2, pp. 143-153, 2002.

[120] E. Mornet, A. Taillandier, S. Peyramaure et al., "Identification of fifteen novel mutations in the tissue-nonspecific alkaline phosphatase (TNSALP) gene in European patients with severe hypophosphatasia," European Journal of Human Genetics, vol. 6, no. 4, pp. 308-314, 1998.

[121] W. Tesch, T. Vandenbos, P. Roschgr et al., "Orientation of mineral crystallites and mineral density during skeletal development in mice deficient in tissue nonspecific alkaline phosphatase," Journal of Bone and Mineral Research, vol. 18, no. 1, pp. 117-125, 2003.

[122] S. Baumgartner-Sigl, E. Haberlandt, S. Mumm et al., "Pyridoxine-responsive seizures as the first symptom of infantile hypophosphatasia caused by two novel missense mutations (c.677T>C, p.M226T; c.1112C>T, p.T371I) of the tissue-nonspecific alkaline phosphatase gene," Bone, vol. 40, no. 6, pp. 1655-1661, 2007.

[123] R. A. Cahill, D. Wenkert, S. A. Perlman et al., "Infantile hypophosphatasia: transplantation therapy trial using bone fragments and cultured osteoblasts," Journal of Clinical Endocrinology and Metabolism, vol. 92, no. 8, pp. 2923-2930, 2007.

[124] M. P. Whyte, S. Mumm, and C. Deal, "Adult hypophosphatasia treated with teriparatide," The Journal of Clinical Endocrinology and Metabolism, vol. 92, no. 4, pp. 1203-1208, 2007.

[125] M. P. Whyte, W. H. McAlister, L. S. Patton et al., "Enzyme replacement therapy for infantile hypophosphatasia attempted by intravenous infusions of alkaline phosphatase-rich Paget plasma: results in three additional patients," The Journal of Pediatrics, vol. 105, no. 6, pp. 926-933, 1984. 


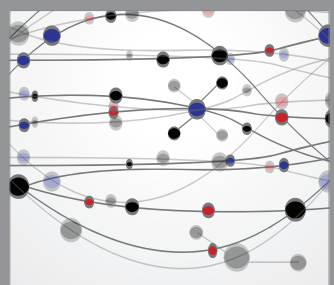

The Scientific World Journal
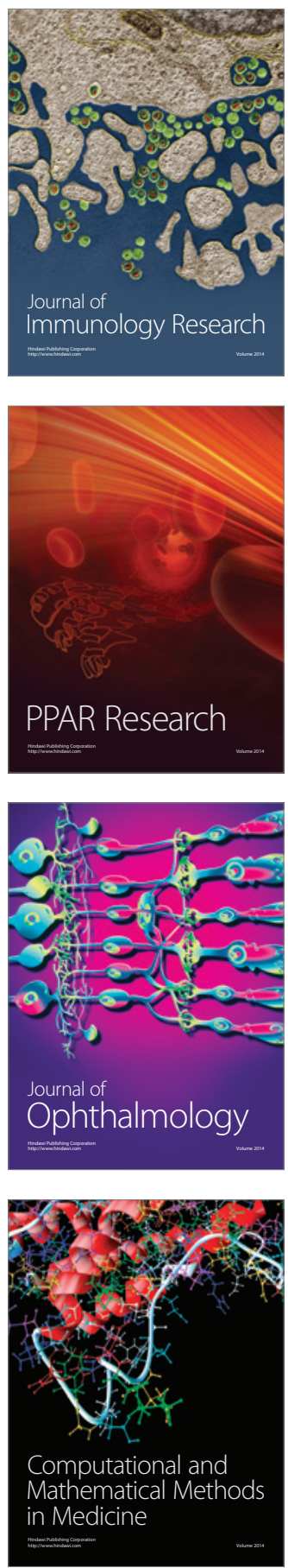

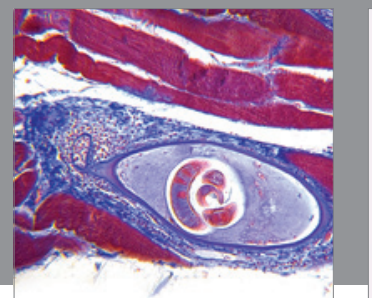

Gastroenterology

Research and Practice
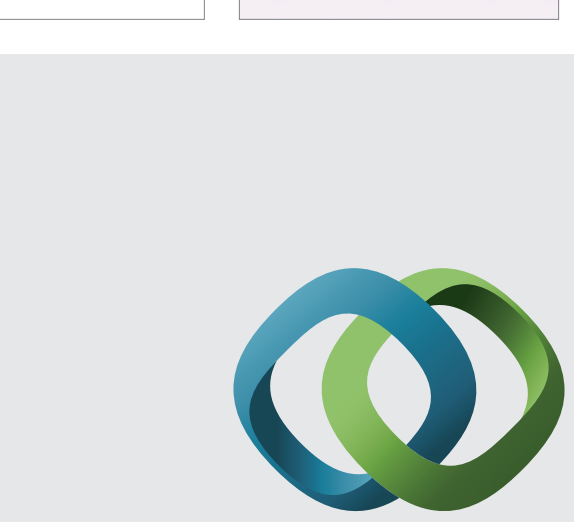

\section{Hindawi}

Submit your manuscripts at

http://www.hindawi.com
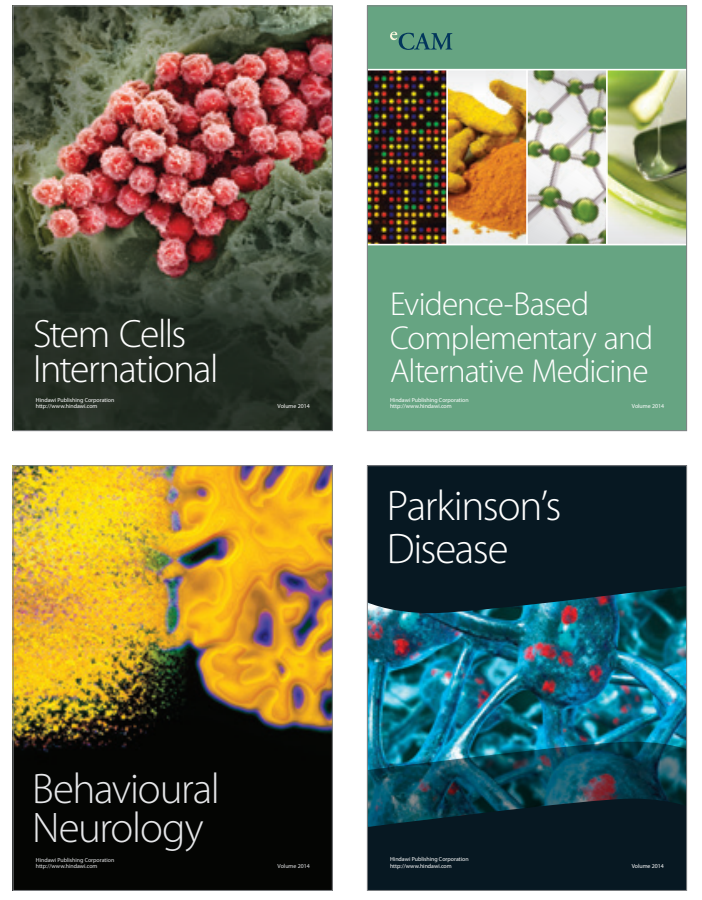
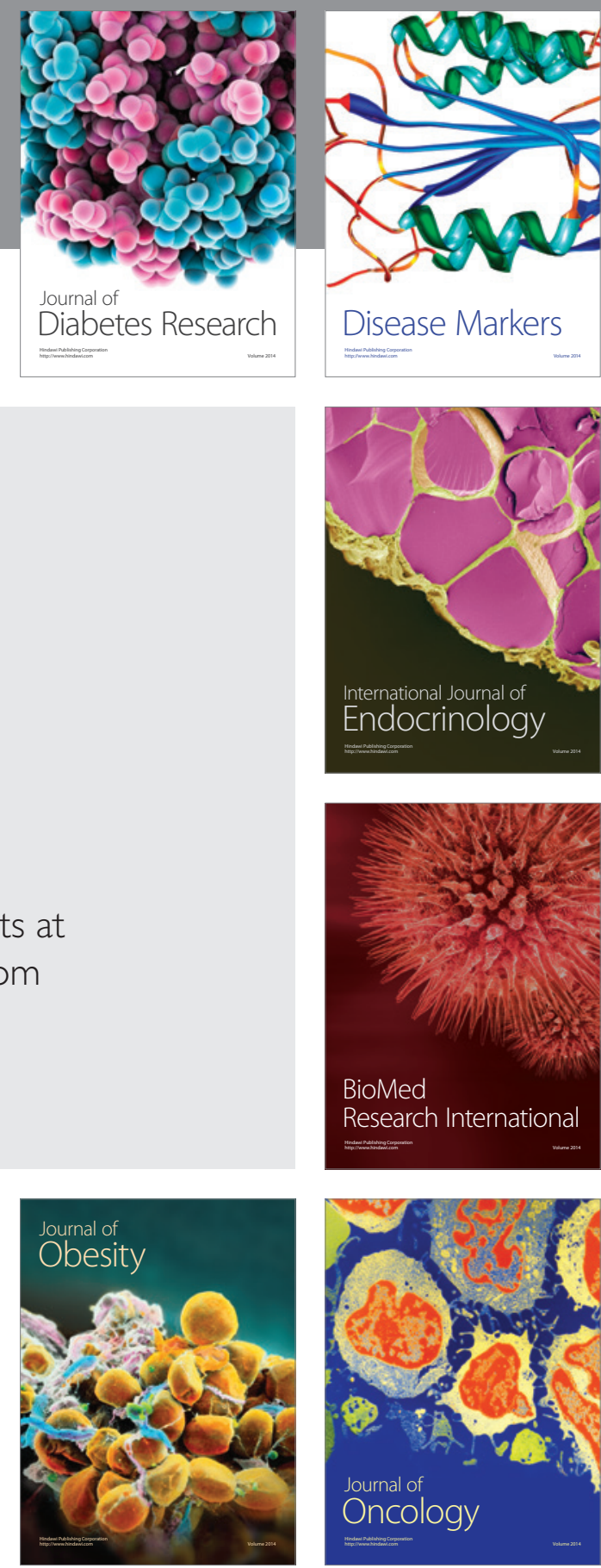

Disease Markers
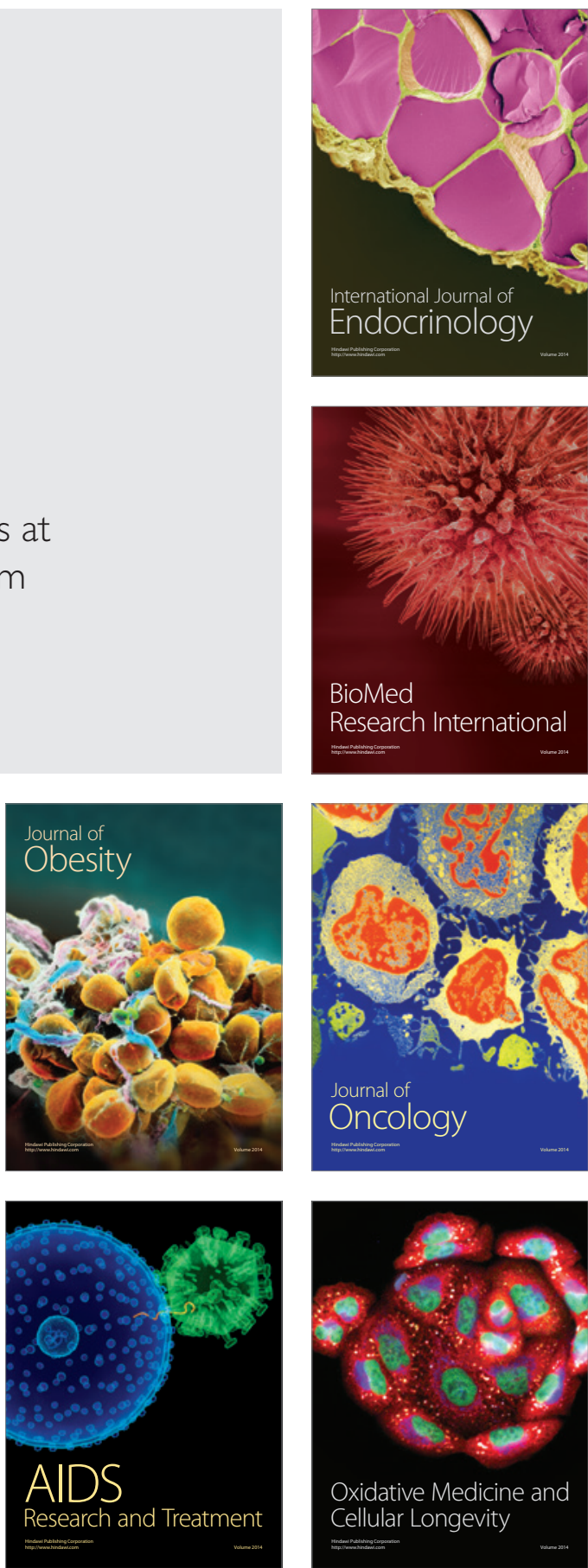